\title{
A Benders decomposition approach for the charging station location problem with plug-in hybrid electric vehicles
}

\author{
Okan Arslan*, Oya Ekin Karaşan \\ Bilkent University, Department of Industrial Engineering, Bilkent, 06800 Ankara, Turkey
}

\section{A R T I C L E I N F O}

\section{Article history:}

Available online 19 September 2016

\section{Keywords:}

Charging station

Location

Flow cover

Benders decomposition

Multicut

Pareto-optimal cuts

Electric vehicles

Plug-in hybrid electric vehicles

\begin{abstract}
A B S T R A C T
The flow refueling location problem (FRLP) locates $p$ stations in order to maximize the flow volume that can be accommodated in a road network respecting the range limitations of the vehicles. This paper introduces the charging station location problem with plug-in hybrid electric vehicles (CSLP-PHEV) as a generalization of the FRLP. We consider not only the electric vehicles but also the plug-in hybrid electric vehicles when locating the stations. Furthermore, we accommodate multiple types of these vehicles with different ranges. Our objective is to maximize the vehicle-miles-traveled using electricity and thereby minimize the total cost of transportation under the existing cost structure between electricity and gasoline. This is also indirectly equivalent to maximizing the environmental benefits. We present an arc-cover formulation and a Benders decomposition algorithm as exact solution methodologies to solve the CSLP-PHEV. The decomposition algorithm is accelerated using Pareto-optimal cut generation schemes. The structure of the formulation allows us to construct the subproblem solutions, dual solutions and nondominated Pareto-optimal cuts as closed form expressions without having to solve any linear programs. This increases the efficiency of the decomposition algorithm by orders of magnitude and the results of the computational studies show that the proposed algorithm both accelerates the solution process and effectively handles instances of realistic size for both CSLP-PHEV and FRLP.
\end{abstract}

(c) 2016 Elsevier Ltd. All rights reserved.

\section{Introduction}

Due to the economic and environmental concerns associated with gasoline, alternative fuel vehicles (AFVs) appeal to customers worldwide. In recent years, a proliferation of AFVs has been observed on the roads (U.S. Department of Energy, 2014). Liquefied petroleum gas (LPG), natural gas, hydrogen, electric and plug-in hybrid electric vehicles are some of the technologies that depend on some form of fuel, different than petroleum, to run. Several parties benefit from their introduction into the transportation sector. From the individual drivers' perspective, they are an efficient way of reducing the transportation costs and environmental impacts such as greenhouse gases (Arslan et al., 2014; U.S. Department of Energy, 2015a; 2015b; Windecker and Ruder, 2013). From the entrepreneurs' perspective, the vehicles themselves as well as the infrastructure they require are possible investment areas. For the oil-importing governments, the AFVs mean less dependence on export oil and governments (e.g., United States) are encouraging fuel provider fleets to implement petroleum-reduction measures (Congress, 2005).

\footnotetext{
* Corresponding author.

E-mail address: okan.arslan@bilkent.edu.tr (0. Arslan).
} 
As the AFVs are known for their rather limited range, their increasing numbers naturally raise the problem of insufficient alternative refueling stations. Lack of enough refueling infrastructure has been identified as one of the barriers for the adoption of AFVs (Bapna et al., 2002; Kuby and Lim, 2005; Melaina and Bremson, 2008; Melaina, 2003; Romm, 2006). In this respect, the refueling station location problem has been touted in the recent literature. There are two mainstream approaches in AFV refueling station location: maximum-coverage and set-covering. The cover-maximization approach for refueling station problem has been considered by Kuby and Lim (2005) with the flow refueling location problem (FRLP). The objective of the FRLP is to locate $p$ stations in order to maximize the flow volume that can be refueled respecting the range limitations of the vehicles. A demand is assumed to be the vehicle flow driving on the shortest path between an origin and destination (OD) pair on a roundtrip. Satisfying a demand, or in other words, refueling a flow requires locating stations at a subset of the nodes on the path such that the vehicles never run out of fuel. Kuby and Lim (2005) pregenerate minimal feasible combinations of facilities to be able to refuel a path, and then build a mixed integer linear programming (MILP) problem to solve the FRLP. There are applications of the problem in the literature (Kuby et al., 2009), analyses are carried out to better understand the driver behavior (Kuby et al., 2013) and different extensions to the original problem are considered such as capacitated stations (Upchurch et al., 2009), driver deviations from the shortest paths (Kim and Kuby, 2012; Ylldiz et al., 2015) and locating stations on arcs (Kuby and Lim, 2007). Different than other studies on FRLM, Kuby et al. (2009) consider maximizing the vehicle-miles-traveled (VMT) on alternative fuel rather than maximizing the flow volume. The authors use FRLM for the location decisions of hydrogen stations in Florida and build a decision support system to investigate strategies for setting up an initial refueling infrastructure in the metropolitan Orlando and statewide. Since the pregeneration phase of the method by Kuby and Lim (2005) requires extensive memory and time, several solution enhancements have been proposed (Capar and Kuby, 2012; Capar et al., 2013; Kim and Kuby, 2013; Lim and Kuby, 2010; MirHassani and Ebrazi, 2013). In particular, MirHassani and Ebrazi (2013) present an innovative graph transformation and Capar et al. (2013) propose a novel modeling logic for the FRLP both of which increase the solution efficiency of the FRLP drastically. In the set-covering approach to the refueling station location problem, the objective is to minimize the number of stations while covering every possible demand in the network (Li and Huang, 2014; MirHassani and Ebrazi, 2013; Wang and Lin, 2009; Wang and Wang, 2010).

FRLM and flow covering problems in general have recently been used in the literature to locate charging stations (Chung and Kwon, 2015; Hosseini and MirHassani, 2015; Jochem et al., 2015; Wang, 2011; Wang and Lin, 2009; 2013). Along with the ideas behind all these applications of flow based models to the charging station location problem, Nie and Ghamami (2013) identified Level 3 fast charging as necessary to achieve a reasonable level of service in intercity charging station location. A similar result is also attained in Lin and Greene (2011) using the National Household Travel Survey. Therefore, similar to aforementioned studies, we assume that the charging stations to be located provide Level 3 service.

In this study, our objective is to embed the plug-in hybrid electric vehicles (PHEVs) into the charging station location problem. All of the aforementioned studies related to refueling or charging station location consider single-type-fueled vehicles. However, using dual sources of energy, PHEVs utilize electricity as well as gasoline for transportation.

Even though PHEVs penetrated the market after HEVs and EVs, they have comparable sales numbers. The sales of PHEVs increased faster than EVs globally in 2015 (Irle et al., 2016) and experts estimate that PHEV sales will surpass EVs in the short term (Shelton, 2016). In 2015, total PHEV sales in Europe and US were 194.615 and 193.757, respectively (Pontes, 2016; U.S. Department of Energy, 2016b). Among top selling PHEVs in US are Chevrolet Volt, Toyota Prius, Ford Fusion and Ford C-Max forming approximately 95\% of the US PHEV market. Mitsubishi Outlander, BYD Qin, BMW i3 are the top three selling brands in Europe sharing more than half of the European PHEV market. Shelton (2016) reports that the sales of EVs and PHEVs are expected to reach to 1 million and 1.35 million in 2020, respectively. Furthermore, PHEV global sales are expected to double by 2025, reaching to 2.7 million PHEVs on the roads.

Similar to the approach by Kuby et al. (2009), we maximize the VMT on electricity which minimizes the total cost of transportation under the existing cost structure between electricity and gasoline. Even though maximizing the AFV numbers in long-distance trips brings environmental benefits, maximizing VMT brings along additional value from an environmental point of view; and in essence, it is also equivalent to minimizing the effects of greenhouse gases. In this context, green transportation is an emerging research topic that has made its debut in the literature in recent years. There are different studies taking into account the green perspective in transportation problems and considering the mileage driven on electricity such as green vehicle routing problem (Erdoğan and Miller-Hooks, 2012; Schneider et al., 2014 ) and optimal routing problems (Arslan et al., 2015). With our approach to the charging station location problem, the environmental benefits of charging station location are fully exploited by considering VMT and additionally taking the PHEVs into account.

\subsection{Contributions}

We introduce the charging station location problem with plug-in hybrid electric vehicles as a generalization of the flow refueling location problem by Kuby and Lim (2005). To our knowledge, this is the first study to consider the PHEVs in intercity charging station location decisions. We minimize the total cost of transportation by maximizing the total distance traveled using electricity. We also address the topic of multi-class vehicles with different ranges in our formulations, which has been discussed as a future research topic in several studies starting with Kuby and Lim (2005). For the exact solution of this practically important and theoretically challenging problem, we present an arc-cover model. To enhance the solution process, we propose a Benders decomposition (BD) algorithm. We construct subproblem solutions in closed form expres- 
sions to accelerate the algorithm. Furthermore, three different cut generation schemes are proposed: singlecut, multicut and Pareto-optimal cut. Using the special structure of the subproblem and its dual, we construct these three types of cuts as closed form expressions without having to solve any LPs. The computational gains with the Pareto-optimal cut generation scheme are significant.

In the following section, the problem is formally introduced. The formulation and the BD algorithm are presented in Sections 3 and 4, respectively. The computational results and the accompanying discussion follow in Section 5 . We conclude the study in Section 6.

\section{The charging station location problem with plug-in hybrid electric vehicles}

Similar to MirHassani and Ebrazi (2013), we start with a brief discussion on the common sense in charging logic, formally introduce the charging station location problem with plug-in hybrid electric vehicles (CSLP-PHEV) and present its complexity status.

\subsection{Common sense in charging logic}

Charging logic and range of EVs: The AFVs have a limited range and they need to refuel on their way to the destination before running out of fuel. Taking the limited range into account, Kuby and Lim (2005) extensively discuss the refueling logic of AFVs. In their study, the AFVs are assumed to have their tank half-full at the origin node and they are required to have at least half-full tank when arriving at the destination node unless a refueling station is located at these nodes. This ensures that they can make their trip back to the same refueling station again in the following trip. The charging logic of EVs is similar to the refueling logic of AFVs with only one minor difference: they can be charged at their origin and destination nodes since these nodes represent cities and dense residential areas where there exist charging opportunities possibly at the drivers' home, at shopping malls, parking lots or at charging stations. Hence, we assume that the driver has fully charged battery at the origin node, and it is allowed to arrive at the destination node with a depleted battery. When traveling intercity, the EVs require charging stations on the road to facilitate the trip. In this study, we consider the location of such intercity charging facilities. Note that this is not a restrictive assumption from the methodological perspective. The means to adapt the model for different charging logics without affecting our solution methodology is discussed in the following section.

Charging logic and range of PHEVs: Using its internal combustion engine, a PHEV can travel on gasoline similar to a conventional vehicle. It can also charge its battery at a charging station and travel using electricity until a minimum state of charge is reached, similar to an EV. Existing studies suggest that PHEV drivers actively search for electricity usage opportunities to avoid the use of gasoline (He et al., 2013; Lin and Greene, 2011). A recent survey carried out by Axsen and Kurani (2008) reveals that early PHEV consumers are generally enthusiastic about any available opportunity to charge their batteries (Lin and Greene, 2011). Considering the price effect of gasoline on the consumer behavior (Walsh et al., 2004; Weis et al., 2010), He et al. (2013) also point out that PHEVs can benefit from the available charging opportunities due to the cost difference between gasoline and electricity. With the sparsely located charging stations especially in the initial stages of infrastructure establishment, we also assume PHEVs will stop at available charging stations to decrease transportation costs. One way of relaxing this strong assumption will be discussed in the following section.

One-way trip: When considering the connectivity of an origin-destination pair, similar to FRLP, we consider the shortest path between the OD pairs. With our origin and destination charging availability assumption, considering only the one-way trip is without loss of generality. Note that for EVs, enabling this trip ensures that the round-trip between the same OD pair is also feasible. On the PHEV side, consider two consecutive nodes that a PHEV stops for charging. Regardless of the direction that the PHEV is traveling between these two nodes, the distance that it can travel on electricity is the same on this connection. Thus, enabling a one-way trip also ensures the round trip feasibility for PHEVs.

\subsection{Problem definition}

Definition 1. Let $G=(N, E)$ represent our transportation network where $N$ is the set of nodes and $E$ is the set of edges. Let $A=\{(i, j) \cup(j, i):\{i, j\} \in E\}$ be the set of directional arcs implied by edges in $E$. An EV demand $q$ is a four-tuple $\left\langle s(q), t(q), f_{e v}^{q}, L_{e v}\right\rangle$, where $s(q), t(q) \in N$ are the origin and the destination nodes of the demand, respectively; $f_{e v}^{q}$ is the EV flow traveling on the shortest path between $s(q)$ and $t(q)$; and $L_{e v}$ is the electric range of the particular EV. A PHEV demand $q=\left\langle s(q), t(q), f_{\text {phev }}^{q}, L_{\text {phev }}\right\rangle$, is similarly defined.

The sets of EV and PHEV demands are referred to as $Q^{e v}$ and $Q^{\text {phev }}$, respectively. The demand set is $Q=Q^{e v} \cup Q^{\text {phev }}$.

Definition 2. A CSLP-PHEV instance is a four tuple $\langle G, Q, K, p\rangle$ where $G$ is the transportation network, $Q$ is the set of demands, $K \subseteq N$ is the set of candidate nodes, and $p \leq|K|$ is a positive integer. Given such an instance, the CSLP-PHEV problem is defined as finding the set of nodes with cardinality $p$ such that the distance to be covered on electricity is maximized. 
(a)

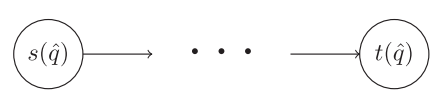

(b)

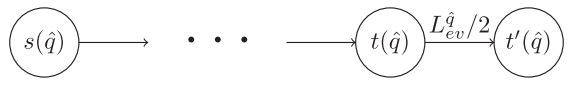

Fig. 1. Network transformation to relax full charge assumption at the destination node.

\subsection{Complexity}

\section{Proposition 1. CSLP-PHEV is NP-Complete.}

Proof. Given an CSLP-PHEV instance, it is easy to check the feasibility of the problem. Thus, CSLP-PHEV is in NP. In order to show that CSLP-PHEV is NP-Complete, we now provide a transformation from the FRLP. Consider an FRLP instance $\langle G, F, K$, $p\rangle$, with network $G$, demand set $F$, candidate facility nodes $K \subseteq N$, and the number of stations to be located $p$. For the CSLPPHEV instance $\left\langle G^{\prime}, Q^{p h e v} \cup Q^{e v}, K, p\right\rangle$, let $Q^{p h e v}=\emptyset$, and $Q^{e v}=F$ with $f_{e v}^{q}=f_{a f v}^{q} / d^{q}$ where $f_{a f v}^{q}$ is the flow of alternative fuel vehicles and $d^{q}$ is the shortest distance between $s(q)$ and $t(q)$. For the corresponding network $G^{\prime}$, we add two dummy nodes $s^{\prime}(q)$ and $t^{\prime}(q)$ for each $q \in F$, and two dummy arcs $\left(s^{\prime}(q), s(q)\right)$ and $\left(t(q), t^{\prime}(q)\right)$ with lengths of $L_{e v} / 2$ to $G$. Observe that solving this CSLP-PHEV instance is equivalent to solving the corresponding FRLP instance. Thus, CSLP-PHEV is NP-Complete.

\subsection{Relaxing our assumptions for different charging logics}

In this subsection, we will identify possible remedies for relaxing the major assumptions of the charging logics as presented above. The first assumption is related to the charging logic of PHEVs. We assume that all PHEV drivers stop to charge whenever possible. Though, this is a very strong assumption, our approach can be adapted to deal with it. In particular, assume that only a fraction of these drivers, say $v$ percent, prefer stopping to charge while the remaining drivers simply prefer to keep driving on gasoline. Observe that the location of the charging stations will not be affected by those drivers who are intolerant to stopping. Therefore, we can simply take $v \%$ of the PHEV drivers into account when constructing our problem instances.

The second major assumption is regarding the availability of the charging stations at the destination nodes. Observe that, without sufficient infrastructure in place, this assumption might not generally hold, and not all destinations might have a charging station available. However, this assumption can easily be relaxed by a simple network transformation as presented in Fig. 1. Let $\hat{q}$ be an EV demand driving between nodes $s(\hat{q})$ and $t(\hat{q})$. If we assume full charge availability at the destination node, then there is no need for a transformation and the network can be taken as in part (a) in the figure. If we assume that there is no charging station at the destination node, then we need to make sure that the EV arrives at the destination node half-full charged. This charge level is to enable a trip back to the last-visited charging station. Similar reasoning is also applied in FRLM by Kuby and Lim (2005). To ensure that the EV arrives at the destination node with a half-full charge, we add a dummy node $t^{\prime}(\hat{q})$ and an $\operatorname{arc}$ between nodes $t(\hat{q})$ and $t^{\prime}(\hat{q})$ with a distance of $L_{e v} / 2$. After the transformation the new destination of the EV demand $\hat{q}$ is now $t^{\prime}(\hat{q})$, as in part (b) in the figure. Similarly, one can relax the full charge assumption at the origin node.

\section{Mathematical model}

In this section, the required notation and the charging station location model with plug-in hybrid electric vehicles (CSLMPHEV) are presented. Given an CSLP-PHEV instance, let $N^{q} \subseteq N$ and $A^{q} \subseteq A$ be the sets of nodes and arcs, respectively, on the shortest path between $s(q)$ and $t(q), \forall q \in Q$. Let $d_{a}, \forall a \in A^{q}$ be the arc distance and $d^{q}=\sum_{a \in A^{q}} d_{a}$ be the distance of the shortest path. Now, for a given $q \in Q^{e v}$, consider an arc $a \in A^{q}$, and a node $i \in N^{q}$ appearing on the shortest path prior to traversing arc $a$. Observe that if the distance between node $i$ and head node of arc $a$ on the shortest path between $s(q)$ and $t(q)$ is less than or equal to $L_{e v}$, then an EV can charge at node $i$ and traverse arc $a$ completely. In this context, let $K_{a}^{q}$ be the set of nodes $i \in K$ that enable complete traversal of the directional arc $a \in A^{q}$ by an EV. For a given $q \in Q^{\text {phev }}$, partial traversal of an arc is also plausible. Let $d_{a}^{q i}$ be the distance on arc $a \in A^{q}$ that can be traveled by a PHEV using electricity if there exists a charging station at node $i \in N^{q}$, and let $R_{a}^{q}$ be the set of candidate sites that enable at least partial traversal of 
the directional arc $a \in A^{q}$ by a PHEV using electricity. We need the following decision variables:

$$
\begin{aligned}
& x_{k}= \begin{cases}1, & \text { if a refueling station is located at node } k \in K \\
0, & \text { otherwise }\end{cases} \\
& z_{q}= \begin{cases}1, & \text { if EV demand } q \text { is covered } \\
0, & \text { otherwise }\end{cases}
\end{aligned}
$$

$y_{a}^{q i}= \begin{cases}1, & \text { if PHEV demand on arc } a \text { travels at least partially on electricity by charging at node } i \\ 0, & \text { otherwise }\end{cases}$

CSLM-PHEV formulation is as follows:

$$
\text { maximize } \sum_{q \in Q^{\text {ev }}} f_{e v}^{q} d^{q} z^{q}+\sum_{\substack{q \in \mathcal{Q P}^{\text {phev }} \\ a \in A^{q} \\ i \in R_{a}^{q}}} f_{\text {phev }}^{q} d_{a}^{q i} y_{a}^{q i}
$$

subject to

$$
\begin{aligned}
& y_{a}^{q i} \leq x_{i} \quad \forall q \in Q^{\text {phev }}, a \in A^{q}, i \in R_{a}^{q} \backslash s(q) \\
& \sum_{i \in R_{a}^{q}} y_{a}^{q i} \leq 1 \quad \forall q \in Q^{p h e v}, a \in A^{q} \\
& z^{q} \leq \sum_{i \in K_{a}^{q}} x_{i} \quad \forall q \in Q^{e v}, a \in A^{q} \\
& \sum_{k \in K} x_{k}=p \\
& x_{k} \in\{0,1\} \quad \forall k \in K \\
& y_{a}^{q i} \in\{0,1\} \quad \forall q \in Q^{p h e v}, a \in A^{q}, i \in R_{a}^{q} \\
& z^{q} \in\{0,1\} \quad \forall q \in Q^{e v}
\end{aligned}
$$

The objective function maximizes the distance traveled using electricity by both EVs and PHEVs. Constraints (2) ensure that if the PHEV associated with demand $q$ travels using electricity on an arc $a$ by refueling at node $i$, then node $i$ must have a charging station except when $i=s(q)$ due to the charging logic. Constraints (3) make sure that an arc $a$ is not counted more than once in the objective function even if more than one station is capable of refueling the vehicle's travel over that arc. Constraints (4) are for setting $z^{q}$ equal to 1 only if all of the path between $s(q)$ and $t(q)$ is traversable using electricity by the EV associated with demand $q$. These constraints are inherited from the study by Capar et al. (2013). Constraints (5) set the number of open facilities to $p$. Constraints (6)-(8) are domain restrictions.

\subsection{Variable relaxation}

Proposition 2. The variables $y_{a}^{q i}$ and $z^{q}$ necessarily assume binary values in an extreme point of the polyhedron that is formed by relaxing their integrality requirement in the CSLM-PHEV formulation.

Proof. Let $\overline{\text { CSLM-PHEV-R }}$ be the polyhedron that is formed by relaxing the $y_{a}^{q i}$ and $z^{q}$ variables in the CSLM-PHEV formulation. Now, assume that there exists an extreme point of CSLM-PHEV-R, say $\xi=(x ; y ; z)$, with some fractional entries in $y$. For a given $\hat{q} \in Q^{\text {phev }}$ and $\hat{a} \in A^{q}$, let $T_{\hat{a}}^{\hat{q}}=\left\{i \in R_{\hat{a}}^{\hat{q}}: 0<y_{\hat{a}}^{\hat{q} \hat{i}}<1\right\}$. Consider the unit vector $e^{i}$ (of length $\left|R_{a}^{q} \backslash s(q)\right|$ with $i^{\text {th }}$ entry equal to 1 and the remaining entries equal to 0 ), and the zero vector $\mathbf{0}$ (of length $\left|R_{a}^{q} \backslash s(q)\right|$ with all entries equal to zero). Note that $(x ; \mathbf{0} ; z),\left(x ; e^{i} ; z\right) \in \overline{\text { CSLM-PHEV-R }}, \forall i \in T_{\hat{a}}^{\hat{q}}$ due to Constraints (2) and (3). Observe that the point $\xi$ can be represented as a strict convex combination of $\left(x ; e^{i} ; z\right)$ and the vector $(x ; \mathbf{0} ; z)$. Thus $\xi$ cannot be an extreme point and $y$ variables necessarily assume binary values. With a similar reasoning, it is easy to see that the $z$ variables also assume binary values in a solution of CSLM-PHEV-R.

The integrality property of the flow variables has been discussed in the literature starting with the introduction of the original FRLM problem by Kuby and Lim (2005).

\subsection{Variable elimination}

For a given $q \in Q$, the set $A^{q}$ contains all the arcs on the shortest path between $s(q)$ and $t(q)$ and the number of variables and constraints depends on the size of this set. However, due to the charging logic of EVs and PHEVs, the size of the set 
$A^{q}$ can be significantly reduced. Let $B_{e v}^{q}$ and $B_{\text {phev }}^{q}$ be the sets of arcs on the shortest path between $s(q)$ and $t(q)$ that can be completely covered by an EV or PHEV, respectively, by charging at the origin node (i.e. the distance between the origin node and the head node of an arc in sets $B_{e v}^{q}$ and $B_{\text {phev }}^{q}$ is less than the range of EV and PHEV, respectively). Since we assume that the EVs and PHEVs begin their trip fully charged at the origin node, the arcs in sets $B_{e v}^{q}$ and $B_{p h e v}^{q}$ can always be completely traversed using electricity.

Let $A_{e v}^{q}$ and $A_{\text {phev }}^{q}$ be the sets of arcs on the shortest path between $s(q)$ and $t(q)$ excluding $B_{e v}^{q}$ and $B_{\text {phev }}^{q}$, respectively. More formally, $A_{e v}^{q}=\left\{a \in A^{q}: a \notin B_{e v}^{q}\right\}$ and $A_{\text {phev }}^{q}=\left\{a \in A^{q}: a \notin B_{p h e v}^{q}\right\}$. By considering these sets rather than the set $A^{q}$, variables $y_{a}^{q i}, \forall q \in Q^{p h e v}, a \in B_{\text {phev }}^{q}, i \in R_{a}^{q}$ are eliminated from the formulation, and the number of Constraints (2)-(4) are reduced. Then, the final formulation, which we shall refer to as CSLM-PHEV becomes:

maximize $\sum_{q \in Q^{\text {ev }}} f_{e v}^{q} d^{q} z^{q}+\sum_{\substack{q \in Q^{\text {phev }} \\ a \in A_{\text {phev }}^{q} \\ i \in R_{a}^{q}}} f_{\text {phev }}^{q} d_{a}^{q i} y_{a}^{q i}+\sum_{\substack{q \in Q^{\text {phev }} \\ a \in B_{\text {phev }}^{q}}} f_{\text {phev }}^{q} d_{a}$

subject to

$y_{a}^{q i} \leq x_{i} \quad \forall q \in Q^{p h e v}, a \in A_{\text {phev }}^{q}, i \in R_{a}^{q} \backslash s(q)$

$\sum_{i \in R_{a}^{q}} y_{a}^{q i} \leq 1 \quad \forall q \in Q^{p h e v}, a \in A_{\text {phev }}^{q}$

$z^{q} \leq \sum_{i \in K_{a}^{q}} x_{i} \quad \forall q \in Q^{e v}, a \in A_{e v}^{q}$

$\sum_{k \in K} x_{k}=p$

$z^{q} \leq 1 \quad \forall q \in Q^{e v}$

$y_{a}^{q i} \geq 0 \quad \forall q \in Q^{p h e v}, a \in A_{\text {phev }}^{q}, i \in R_{a}^{q}$

$z^{q} \geq 0 \quad \forall q \in Q^{e v}$

$x_{k} \in\{0,1\} \quad \forall k \in K$

Let $\mathcal{F}=\sum_{\substack{q \in Q^{p h e v} \\ a \in B_{\text {phev }}^{q}}} f_{\text {phev }}^{q} d_{a}$, as it appears in the objective function. Since $\mathcal{F}$ is fixed for a given CSLM-PHEV instance, we exclude it from the formulations in the following parts of the paper for conciseness. Note that, a covered arc contributes to the objective function for the PHEVs, but not for the EVs. Therefore, there does not exist a fixed term in the objective function related to EVs and the set $B_{e v}^{q}$. If all of the arcs on a given path of $\mathrm{EV}$ demand $\hat{q} \in Q^{e v}$ can be covered by charging at the origin, then Constraints (12) are eliminated for $\hat{q}$ in the formulation and $z_{\hat{q}}$ variable naturally assumes a value of 1.

\section{Benders decomposition}

Considering PHEVs beside EVs further compound the challenge in the charging station location problem. There are several successful implementations of Benders decomposition (Benders, 1962) for location problems in the recent literature (de Camargo et al., 2008; 2009; Contreras et al., 2011; Cordeau et al., 2000; 2001; Costa, 2005; Fontaine and Minner, 2014; Froyland et al., 2013; Khatami et al., 2015; Martins de Sá et al., 2015; de Sá et al., 2013; Wheatley et al., 2015; Wu et al., 2005). In this section, we propose a BD algorithm as the solution technique and apply enhancements to improve the solution time of the algorithm by efficiently solving the subproblem and constructing Pareto-optimal cuts in closed form expressions. Observe that in our particular case, fixing the location variables $x$ turns the formulation into a linear programming model due to Proposition 2. In our presentation, we use a similar notation to the study by Üster and Kewcharoenwong (2011).

\subsection{Benders subproblem}

For a given $\hat{x} \in\{0,1\}^{|K|}$, the subproblem of the CSLM-PHEV, referred to as $\operatorname{SP}(y, z \mid \hat{x})$ is presented below:

$$
\text { maximize } \sum_{q \in Q^{e v}} f_{e v}^{q} d^{q} z^{q}+\sum_{\substack{q \in Q^{\text {phev }} \\ a \in A_{\text {phev }}^{q} \\ i \in R_{a}^{q}}} f_{\text {phev }}^{q} d_{a}^{q i} y_{a}^{q i}
$$


subject to

$$
\begin{aligned}
& y_{a}^{q i} \leq \hat{x}_{i} \quad \forall q \in Q^{\text {phev }}, a \in A_{\text {phev }}^{q}, i \in R_{a}^{q} \backslash s(q) \\
& \sum_{i \in R_{a}^{q}} y_{a}^{q i} \leq 1 \quad \forall q \in Q^{\text {phev }}, a \in A_{\text {phev }}^{q} \\
& z^{q} \leq \sum_{i \in K_{a}^{q}} \hat{x}_{i} \quad \forall q \in Q^{e v}, a \in A_{e v}^{q} \\
& z^{q} \leq 1 \quad \forall q \in Q^{e v} \\
& y_{a}^{q i} \geq 0 \quad \forall q \in Q^{\text {phev }}, a \in A_{\text {phev }}^{q}, i \in R_{a}^{q} \\
& z^{q} \geq 0 \quad \forall q \in Q^{e v}
\end{aligned}
$$

Note that setting all the variables equal to zero is a feasible solution to the subproblem. Due to Constraints (19)-(22), it is also bounded.

Let $\mu, \rho, \phi$ and $\sigma$ be the dual variables associated with constraints (19), (20), (21) and (22), respectively. Then, the dual subproblem, referred to as $\operatorname{SPD}(\mu, \rho, \phi, \sigma \mid \hat{x})$, is expressed as follows:

$$
\text { minimize } \sum_{\substack{q \in Q^{\text {phev }} \\ a \in \rho^{q} \\ i \in R_{a}^{p h e v}}} \hat{x}_{i} \mu_{a}^{q i}+\sum_{\substack{q \in Q^{\text {phev }} \\ a \in A_{\text {phev }}^{q}}} \rho_{a}^{q}+\sum_{\substack{q \in Q^{e v} \\ a \in A_{e v}^{q} \\ i \in K_{a}^{q}}} \hat{x}_{i} \phi_{a}^{q}+\sum_{q \in Q^{e v}} \sigma_{q}
$$

subject to

$$
\begin{aligned}
& \sum_{a \in A_{e v}^{q}} \phi_{a}^{q}+\sigma_{q} \geq f_{e v}^{q} d^{q} \quad \forall q \in Q^{e v} \\
& \mu_{a}^{q i}+\rho_{a}^{q} \geq f_{\text {phev }}^{q} d_{a}^{q i} \quad \forall q \in Q^{\text {phev }}, a \in A_{\text {phev }}^{q}, i \in R_{a}^{q} \backslash s(q) \\
& \rho_{a}^{q} \geq f_{\text {phev }}^{q} d_{a}^{q s(q)} \quad \forall q \in Q^{\text {phev }}, a \in A_{\text {phev }}^{q}, s(q) \in R_{a}^{q} \\
& \mu_{a}^{q i}, \rho_{a}^{q} \geq 0 \quad \forall q \in Q^{\text {phev }}, a \in A_{\text {phev }}^{q}, i \in R_{a}^{q} \backslash s(q) \\
& \phi_{a}^{q}, \sigma_{q} \geq 0 \quad \forall q \in Q^{e v}, a \in A_{e v}^{q}
\end{aligned}
$$

\subsection{Benders master problem}

Let $\mathcal{D}(S P D)$ denote the set of extreme points of $\operatorname{SPD}(\mu, \rho, \phi, \sigma \mid \hat{x})$. Then, we can construct the master problem, referred to as MP, as follows:

maximize $\eta$

subject to

$$
\begin{aligned}
& \eta \leq \sum_{\substack{q \in Q^{\text {phev }} \\
a \in A_{\text {phev }}^{q} \\
i \in R_{a}^{q} \backslash S(q)}} x_{i} \mu_{a}^{q i}+\sum_{\substack{q \in Q_{\text {phev }}^{\text {phev }} \\
a \in A_{\text {phev }}^{q}}} \rho_{a}^{q}+\sum_{\substack{q \in Q^{e v} \\
a \in A_{e v}^{q v} \\
i \in K_{a}^{q}}} x_{i} \phi_{a}^{q}+\sum_{q \in Q^{e v}} \sigma_{q} \quad \forall(\mu, \rho, \phi, \sigma) \in \mathcal{D}(S P D) \\
& \sum_{k \in K} x_{k}=p \\
& x_{k} \in\{0,1\} \quad \forall k \in K
\end{aligned}
$$

We have now transformed the CSLM-PHEV model into an equivalent mixed integer programming model with $|K|$ binary and one continuous variables. In order to handle the exponential number of constraints of the MP model due to the set $\mathcal{D}(S P D)$, we apply a branch-and-cut approach. Using the dual variable values obtained from the subproblem, a cut is added to the master problem at each iteration in the form of an inequality (32). Note that since the primal subproblem is always feasible and bounded, by strong duality, the dual is also feasible and bounded. Therefore, the cuts added at each iteration are optimality cuts.

The classical Benders implementation solves the master problem to optimality before solving the subproblem. This entails the possibility of unnecessarily revisiting the same solutions again in the master problem. Therefore, we solve our problem in a single branch and bound tree similar to Codato and Fischetti (2006). At every potential incumbent solution encountered 
in the search tree, we solve the subproblem and add an optimality cut, if necessary. In our computational studies, we implement this method using the lazy constraint callback of the CPLEX Concert Technology.

\subsection{Subproblem solution}

Observe that the subproblem $S P(y, z \mid \hat{x})$ can be decomposed based on the vehicle type. The first term in the objective function and Constraints (21), (22) and (24) are related to EVs, while the remaining parts are related to PHEVs. We refer to the former problem as $S P^{e v}(z \mid \hat{x})$, and the latter as $S P^{p h e v}(y \mid \hat{x})$. Observe that $S P^{e v}(z \mid \hat{x})$ can further be decomposed on the basis of demand. For a given demand $q \in Q^{e v}$, we refer to the partition which is formulated below as $S P_{q}^{e v}(z \mid \hat{x})$.

maximize $f_{e v}^{q} d^{q} z^{q}$

subject to

$$
\begin{aligned}
& z^{q} \leq \sum_{i \in K_{a}^{q}} \hat{x}_{i} \quad \forall a \in A_{e v}^{q} \\
& z^{q} \leq 1 \\
& z^{q} \geq 0
\end{aligned}
$$

Let $K^{\star}(\hat{x})=\left\{k \in K: \hat{x}_{k}=1\right\}$. Observe that identifying if the path between $s(q)$ and $t(q)$ can be traveled by an EV without running out of electricity can be done by checking the existence of an open station to cover every arc on the path. Hence, the following variable construction is an optimal solution for $S P_{q}^{e v}(z \mid \hat{x})$.

$$
z^{q}= \begin{cases}1, & \text { if }\left|K_{a}^{q} \cap K^{\star}(\hat{x})\right| \geq 1, \forall a \in A_{e v}^{q} \\ 0, & \text { otherwise }\end{cases}
$$

As for the PHEVs, the $S P^{p h e v}(y \mid \hat{x})$ problem can be decomposed on the basis of demand and arc. For a given demand $q \in$ $Q^{\text {phev }}$ and $a \in A_{\text {phev }}^{q}$, we refer to the partition which is formulated below as $\operatorname{SP}_{q a}^{\text {phev }}(y \mid \hat{x})$ :

$$
\text { maximize } \sum_{i \in R_{a}^{q}} f_{p h e v}^{q} d_{a}^{q i} y_{a}^{q i}
$$

subject to

$$
\begin{aligned}
& y_{a}^{q i} \leq \hat{x}_{i} \quad \forall i \in R_{a}^{q} \backslash s(q) \\
& \sum_{i \in R_{a}^{q}} y_{a}^{q i} \leq 1 \\
& y_{a}^{q i} \geq 0 \quad \forall i \in R_{a}^{q}
\end{aligned}
$$

Similar to the case with EVs, the solution can easily be constructed. Observe that, for the demand $q$, the distance that can be traveled on arc $a$ using electricity is equal to $\max _{j \in R_{a}^{q} \cap\left\{K^{\star}(\hat{x}) \cup s(q)\right\}}\left\{d_{a}^{q j}\right\}$ in which case the associated variable $y_{a}^{q j}$ assumes a value of 1 . That is,

$$
y_{a}^{q i}= \begin{cases}1, & \text { if } i=\operatorname{argmax}_{j \in R_{a}^{q} \cap\left\{K^{\star}(\hat{x}) \cup s(q)\right\}}\left\{d_{a}^{q j}\right\} \\ 0, & \text { otherwise }\end{cases}
$$

\subsection{Benders cut characterization}

In the dual subproblem $\operatorname{SPD}(\mu, \rho, \phi, \sigma \mid \hat{x})$, variables $\phi$ and $\sigma$ and Constraints (26) and (30) are related to the EVs, while variables $\mu$ and $\rho$ and Constraints (27)-(29) are related to PHEVs. Therefore $\operatorname{SPD}(\mu, \rho, \phi, \sigma \mid \hat{x})$ problem can also be decomposed into two: $\operatorname{SPD}^{e v}(\phi, \sigma \mid \hat{x})$ and $\operatorname{SPD}^{\text {phev }}(\mu, \rho \mid \hat{x})$. Note that the former problem can also be decomposed on the basis of demand. Let the $q^{\text {th }}$ partition of the $\operatorname{SPD}^{e v}(\phi, \sigma \mid \hat{x})$ problem be defined as $\operatorname{SPD}_{q}^{e v}(\phi, \sigma \mid \hat{x})$, which is stated as follows:

$$
\text { minimize } \sum_{\substack{a \in A_{e v}^{q} \\ i \in K_{a}^{q}}} \hat{x}_{i} \phi_{a}^{q}+\sigma_{q}
$$

subject to

$$
\sum_{a \in A_{e v}^{q}} \phi_{a}^{q}+\sigma_{q} \geq f_{e v}^{q} d^{q}
$$




$$
\phi_{a}^{q}, \sigma_{q} \geq 0 \quad \forall a \in A_{e v}^{q}
$$

Observe that this is a continuous knapsack problem. Therefore we can construct the solution of this problem in closed form. Before we proceed, we need some further definitions. Let $\mathcal{A}_{0}^{q}(\hat{x})$ and $\mathcal{A}_{1}^{q}(\hat{x})$ be the sets of arcs in $A_{e v}^{q}$, induced by $\hat{x}$, that cannot be covered by any of the open stations and that can only be covered by a single station, respectively. Formally, $\mathcal{A}_{0}^{q}(\hat{x})=\left\{a \in A_{e v}^{q}:\left|K_{a}^{q} \cap K^{\star}(\hat{x})\right|=0\right\}$ and $\mathcal{A}_{1}^{q}(\hat{x})=\left\{a \in A_{e v}^{q}:\left|K_{a}^{q} \cap K^{\star}(\hat{x})\right|=1\right\}$. Note that $\mathcal{A}_{1}^{q}(\hat{x})$ might be empty even if $z_{q}=1$, e.g., if all arcs on the path between $s(q)$ and $t(q)$ can be covered by at least two open stations. But we have a non-empty $\mathcal{A}_{0}^{q}(\hat{x})$ set for any $q$ with $z_{q}=0$.

Proposition 3. For a given $\hat{x}$, and path $\hat{q} \in Q^{e v}$, the following is the characterization of optimal solutions to $S P D_{\hat{q}}^{e v}$.

Case (1) If path $\hat{q}$ is not chargeable (i.e., $z_{\hat{q}}=0$ ), then:

(a) For all $\alpha \in \mathcal{A}_{0}^{\hat{q}}(\hat{x})$, the following solution is optimal:

$$
\begin{aligned}
& -\sigma_{\hat{q}}=0 \\
& -\phi_{\alpha}^{\hat{q}}=f_{e v}^{\hat{q}} d^{\hat{q}} \\
& \text { - } \phi_{a}^{\hat{q}}=0, \forall a \in\left(A_{e v}^{\hat{q}} \backslash \alpha\right) .
\end{aligned}
$$

Case (2) If path $\hat{q}$ is chargeable (i.e., $z_{\hat{q}}=1$ ), then:

(a) For all $\alpha \in \mathcal{A}_{1}^{\hat{q}}(\hat{x})$, the following solution is optimal:

$$
\begin{aligned}
& -\sigma_{\hat{q}}=0 \\
& -\phi_{\alpha}^{\hat{q}}=f_{e v}^{\hat{q}} d \hat{q} \\
& \text { - } \phi_{a}^{\hat{q}}=0, \forall a \in\left(A_{e v}^{\hat{q}} \backslash \alpha\right)
\end{aligned}
$$

(b) The following is also an optimal solution:

$$
\begin{aligned}
& -\sigma_{\hat{q}}=f_{e v}^{\hat{q}} d^{\hat{q}} \\
& -\phi_{a}^{\hat{q}}=0, \forall a \in A_{e v}^{\hat{q}}
\end{aligned}
$$

Proof. All results follow trivially from the fact that $\operatorname{SPD}_{q}^{e v}(\phi, \sigma \mid \hat{x})$ is a continuous knapsack problem.

Note that any convex combination of alternative optimal solutions is also optimal for $\operatorname{SPD}_{q}^{e v}(\phi, \sigma \mid \hat{x})$.

The subproblem for PHEVs which we refer to as $\operatorname{SPD}^{p h e v}(\mu, \rho \mid \hat{x})$ can be decomposed on the basis of demand and arc. We refer to the partition of $a^{\text {th }}$ arc of the $q^{\text {th }}$ demand as $\operatorname{SPD}_{q a}^{\text {phev }}(\mu, \rho \mid \hat{x})$, which is stated as follows:

$$
\text { minimize } \sum_{i \in R_{a}^{q} \backslash s(q)} \hat{x}_{i} \mu_{a}^{q i}+\rho_{a}^{q}
$$

subject to

$$
\begin{aligned}
& \mu_{a}^{q i}+\rho_{a}^{q} \geq f_{\text {phev }}^{q} d_{a}^{q i} \quad \forall i \in R_{a}^{q} \backslash s(q) \\
& \rho_{a}^{q} \geq f_{\text {phev }}^{q} d_{a}^{q s(q)} \quad s(q) \in R_{a}^{q} \\
& \mu_{a}^{q i}, \rho_{a}^{q} \geq 0 \quad \forall i \in R_{a}^{q} \backslash s(q)
\end{aligned}
$$

The following result can be attained through complementary slackness conditions:

Proposition 4. For a given path $\hat{q}$ and $\operatorname{arc} \hat{a} \in A_{\text {phev }}^{\hat{q}}$, the following is the characterization of optimal solutions to $\operatorname{SPD}_{\hat{q} \hat{a}}^{\text {phev }}(\mu, \rho \mid \hat{x})$.

Case (1) If $\sum_{i \in R_{\hat{a}}^{\hat{y}}} \hat{q}_{\hat{a}}^{\hat{q} i}=0$, then the following solution is optimal:

$$
\begin{aligned}
& -\rho_{\hat{a}}^{\hat{q}}=0 \\
& -\mu_{\hat{a}}^{\hat{q} i}=f_{p h e v}^{\hat{q}} d_{\hat{a}}^{\hat{q} i}, \forall i \in R_{\hat{a}}^{\hat{q}} \backslash s(\hat{q})
\end{aligned}
$$

Case (2) If $y_{\hat{a}}^{\hat{q} s(\hat{q})}=1$, then the following solution is optimal: 
$-\rho_{\hat{a}}^{\hat{q}}=f_{\text {phev }}^{\hat{q}} d_{\hat{a}}^{\hat{q} s(\hat{q})}$

$-\mu_{\hat{a}}^{\hat{q} i}=\max \left\{0, f_{\text {phev }}^{\hat{q}} d_{\hat{a}}^{\hat{q} i}-\rho_{\hat{a}}^{\hat{q}}\right\}, \forall i \in R_{\hat{a}}^{\hat{q}} \backslash s(\hat{q})$

Case (3) If $y_{\hat{a}}^{\hat{q} j}=1$ for $j \neq s(\hat{q})$, then the following are the conditions for optimality:

$$
\begin{aligned}
& -\mu_{\hat{a}}^{\hat{q} j}+\rho_{\hat{a}}^{\hat{q}}=f_{\text {phev }}^{\hat{q}} d_{\hat{a}}^{\hat{q} j}, \\
& \text { - } \mu_{\hat{a}}^{\hat{q} i}=0 \text {, and } \rho_{\hat{a}}^{\hat{q}} \geq f_{\text {phev }}^{\hat{q}} d_{\hat{a}}^{\hat{q} i}, \forall i \in R_{\hat{a}}^{\hat{q}}: i \in K^{\star}(\hat{x}), i \neq s(\hat{q}), i \neq j \text {, } \\
& \text { - } \mu_{\hat{a}}^{\hat{q} i}+\rho_{\hat{a}}^{\hat{q}} \geq f_{\text {phev }}^{\hat{q}} d_{\hat{a}}^{\hat{q} i}, \forall i \in R_{\hat{a}}^{\hat{q}}: i \notin K^{\star}(\hat{x}), i \neq s(\hat{q}), i \neq j \text {, } \\
& \text { - } \rho_{\hat{a}}^{\hat{q}} \geq f_{\text {phev }}^{\hat{q}} d_{\hat{a}}^{\hat{q} s(\hat{q})} \text {, if } s(\hat{q}) \in R_{\hat{a}}^{\hat{q}} \text {, } \\
& \text { - } \mu_{\hat{a}}^{\hat{q} i}, \rho_{\hat{a}}^{\hat{q}} \geq 0, \forall i \in R_{\hat{a}}^{\hat{q}}, i \notin K^{\star}(\hat{x}), i \neq s(\hat{q}) \text {. }
\end{aligned}
$$

Proof. Since $\sum_{i \in R_{\hat{a}}^{\hat{q}}} y_{\hat{a}}^{\hat{q} i}=0$, we have $y_{\hat{a}}^{\hat{q} i}=0, \forall i \in R_{\hat{a}}^{\hat{q}}$. We then have $\left|R_{\hat{a}}^{\hat{q}} \cap K^{\star}(\hat{x})\right|=0$, that is $\hat{x}_{i}=0, \forall i \in R_{a}^{q} \backslash s(q)$. Therefore, Case (1) follows. When $\sum_{i \in R_{\hat{a}}^{\hat{q}}} y_{\hat{a}}^{\hat{q} i}=1$, either $y_{\hat{a}}^{\hat{q} s(\hat{q})}=1$ or $y_{\hat{a}}^{\hat{q} j}=1$ for $j \neq s(\hat{q})$. In the former case, we have $d_{\hat{a}}^{\hat{q} s(\hat{q})} \geq d_{\hat{a}}^{\hat{q} i}, \forall i \in$ $R_{\hat{a}}^{\hat{q}}, i \in K^{\star}(\hat{x}), i \neq s(\hat{q})$ since $y_{\hat{a}}^{\hat{q} s(\hat{q})}=1$. Thus, we have $\mu_{\hat{a}}^{\hat{q} j}+\rho_{\hat{a}}^{\hat{q}}=f_{\text {phev }}^{\hat{q}} d_{\hat{a}}^{\hat{q} j}$. This implies that $\mu_{\hat{a}}^{\hat{q} i}=0, \forall i \in R_{\hat{a}}^{\hat{q}}, i \in K^{\star}(\hat{x}), i \neq s(\hat{q})$. For $i \in R_{\hat{a}}^{\hat{q}}, i \notin K^{\star}(\hat{x}), i \neq s(\hat{q})$, we have $\mu_{\hat{a}}^{\hat{q} i}=\max \left\{0, f_{\text {phev }}^{\hat{q}} d_{\hat{a}}^{\hat{q} i}-\rho_{\hat{a}}^{\hat{q}}\right\}$ due to Constraint (49). In the latter case, we have $y_{\hat{a}}^{\hat{q} j}=$ 1 for $j \neq s(\hat{q})$. This implies that $d_{\hat{a}}^{\hat{q} j} \geq d_{\hat{a}}^{\hat{a} i}, \forall i \in R_{\hat{a}}^{\hat{q}}, \forall i \in K^{\star}(\hat{x}), i \neq s(\hat{q}), i \neq j$. Since we have $\hat{x}_{i}=1$ for $i \in R_{\hat{a}}^{\hat{q}}, \forall i \in K^{\star}(\hat{x}), i \neq$ $s(\hat{q}), i \neq j$, we have the first two conditions as given in Case (3). Since we can have $d_{\hat{a}}^{\hat{q} j}<d_{\hat{a}}^{\hat{q} i}$ for $i \in R_{\hat{a}}^{\hat{q}}, i \notin K^{\star}(\hat{x}), i \neq s(\hat{q}), i \neq$ $j$, we keep Constraint (49) in the third bullet of Case (3). The remaining two bullets are also inherited from the constraint set of $\operatorname{SPD}_{q a}^{\text {phev }}(\mu, \rho \mid \hat{x})$. This completes the proof.

Remark 1. Both Propositions 3 and 4 assume primal feasibility as given, and present the properties of optimal solutions for their respective problems.

Remark 2. A Benders cut can be constructed by first solving the subproblem using (39) and (44), and then by generating the dual variables $\mu, \rho, \phi$ and $\sigma$ that satisfy the conditions given in Propositions 3 and 4 . The generated cut can be added to the master problem as an inequality of the form (32).

In the following, we propose three different cut selection schemes. The first one produces a single cut for each subproblem solved. This cut is used as a benchmark in the computational study section to compare the efficiencies of the remaining two cut selection schemes. The next scheme is multicut generation scheme in which several cuts are added at each iteration. In the last scheme, we generate a Pareto-optimal cut.

\subsection{Benders cut selection Scheme 1: single cut}

For a given $\hat{x}$, we can construct the primal subproblem solutions $z^{q}, \forall q \in Q^{e v}$ and $y_{a}^{q i}, \forall q \in Q^{\text {phev }}, a \in A_{\text {phev }}^{q}, i \in R_{a}^{q}$. Consider the corresponding dual solution presented in Algorithm 1 in the Appendix. Parts of the algorithm that are analogous to cases in Propositions 3 and 4 are explicitly shown. The dual variables obtained can be used to construct a single cut to be added to the master problem at each iteration as an inequality of type (32).

\subsection{Benders cut selection Scheme 2: multicut}

Birge and Louveaux (1988) considered addition of several cuts in a single iteration in the context of two-stage stochastic linear programs. Multicut version of BD algorithm has successfully been implemented in different applications (de Camargo et al., 2008; Lei et al., 2014; Trukhanov et al., 2010; You and Grossmann, 2013). To implement the multicut version, we need to modify our master problem. For this purpose, let $\beta_{q}^{e v}$ and $\delta_{q a}^{\text {phev }}$ be new surrogate variables associated with $\operatorname{SPD}_{q}^{e v}(\phi, \sigma \mid \hat{x})$ and $\operatorname{SPD}_{q a}^{\text {phev }}(\mu, \rho \mid \hat{x})$, respectively; $\mathcal{D}\left(\operatorname{SPD}_{q}^{e v}\right)$ and $\mathcal{D}\left(S P D_{q a}^{\text {phev }}\right)$ be the set of extreme points for the respective problems. Then the modified master problem can be expressed as:

$$
\text { maximize } \sum_{q \in Q^{e v}} \beta_{q}^{e v}+\sum_{\substack{q \in Q^{p h e v} \\ a \in A_{\text {phev }}^{q}}} \delta_{q a}^{\text {phev }}
$$


subject to

$$
\begin{aligned}
& \beta_{q}^{e v} \leq \sum_{\substack{a \in A_{e v}^{q} \\
i \in K_{a}^{q}}} x_{i} \phi_{a}^{q}+\sigma_{q} \quad \forall q \in Q^{e v},(\phi, \sigma) \in \mathcal{D}\left(S P D_{q}^{e v}\right) \\
& \delta_{q a}^{p h e v} \leq \sum_{\substack{i \in R_{a}^{q} \backslash s(q) \\
x^{\prime}}} x_{i} \mu_{a}^{q i}+\rho_{a}^{q} \quad \forall q \in Q^{\text {phev }}, a \in A_{p h e v}^{q},(\mu, \rho) \in \mathcal{D}\left(S P D_{q a}^{\text {phev }}\right) \\
& \sum_{k \in K} x_{k}=p \\
& x_{k} \in\{0,1\} \quad \forall k \in K
\end{aligned}
$$

For a given $\hat{x}$, multiple extreme points for each subproblem as presented in Propositions 3 and 4 exist. By modifying the master problem, we can now use the information that is available for each subproblem and add a cut corresponding to each extreme point, as presented in Algorithm 2 in the Appendix. In this fashion, a cut pool is collected at each iteration.

\subsection{Benders cut selection Scheme 3: Pareto-optimal cut}

Reducing the number of Benders iterations is of great value to accelerate the implementation. However, as we have identified above, conditions in Propositions 3 and 4 imply that several cuts can be generated for a given vector $\hat{x}$. Out of these cuts, the simple cut generation scheme selects one cut to be added at each iteration, and the multicut implementation adds several cuts at once. One other option is to select a cut that might help us reduce the number of iterations. In this section, we present the Pareto-optimal cut generation scheme in the sense of the study by Magnanti and Wong (1981). Let $\mathcal{X}$ be the feasible set of the master problem in the first iteration (i.e. $\mathcal{X}=\left\{x \in\{0,1\}^{|K|}: \sum_{k \in K} x_{k}=p\right\}$ ), opt $(\mathcal{P}$ ) be the optimal objective function value of the problem $\mathcal{P}$, and the function $\mathcal{C}(x, \mu, \rho, \phi, \sigma)$ be defined as the following:

$$
\mathcal{C}(x, \mu, \rho, \phi, \sigma)=\sum_{\substack{q \in Q^{\text {phev }} \\ a \in A_{p h e v}^{q} \\ i \in R_{a}^{q} \backslash S(q)}} x_{i} \mu_{a}^{q i}+\sum_{\substack{q \in Q^{\text {phev }} \\ a \in A_{\text {phev }}^{q}}} \rho_{a}^{q}+\sum_{\substack{q \in Q^{e v} \\ a \in A_{e v}^{q} \\ i \in K_{a}^{q}}} x_{i} \phi_{a}^{q}+\sum_{q \in Q^{e v}} \sigma_{q}
$$

In their seminal paper, Magnanti and Wong (1981) presented the notion of cut domination in the BD framework. The cut generated by the dual solution $(\hat{\mu}, \hat{\rho}, \hat{\phi}, \hat{\sigma})$ is said to dominate the cut generated by the dual solution $(\tilde{\mu}, \tilde{\rho}, \tilde{\phi}, \tilde{\sigma})$, if and only if $\mathcal{C}(x, \hat{\mu}, \hat{\rho}, \hat{\phi}, \hat{\sigma}) \leq \mathcal{C}(x, \tilde{\mu}, \tilde{\rho}, \tilde{\phi}, \tilde{\sigma})$ for all $x \in \mathcal{X}$ with a strict inequality for at least one point. A cut is called Paretooptimal, if no other cuts dominate it. To obtain a Pareto-optimal cut induced by $\hat{x}$, we need to solve the following linear programming problem:

$$
\text { minimize } \mathcal{C}(\bar{x}, \mu, \rho, \phi, \sigma)
$$

subject to

$$
\begin{aligned}
& (26)-(30) \\
& \mathcal{C}(\hat{x}, \mu, \rho, \phi, \sigma)=\operatorname{opt}(\operatorname{SPD}(\mu, \rho, \phi, \sigma \mid \hat{x}))
\end{aligned}
$$

where $\bar{x} \in \operatorname{ri}\left(\mathcal{X}^{c}\right)$, a point in the relative interior of the convex hull of the set $\mathcal{X}$, is called a core point. We refer to this LP as $M W(\mu, \rho, \phi, \sigma \mid \hat{x}, \bar{x})$. Our initial core point is a vector of length $|K|$ with all entries equal to $\frac{p}{|K|}$. In the following Benders iterations, we use the average of the current core point and the active solution of the MP, similar to Papadakos (2008).

At this point, note that Constraint (59) and fractional coefficients $\bar{x}_{i}$ may prevent obtaining an efficient optimal-dual solution, as is the case for the study by Contreras et al. (2011). However, as we present in the following parts, we can cleverly exploit the knapsack nature of the subproblems to find the optimal solutions for the MW problem using Remark (1).

\subsection{Magnanti-Wong problem solution}

Similar to the subproblem and the dual of the subproblem, the $M W(\mu, \rho, \phi, \sigma \mid \hat{x}, \bar{x})$ problem can also be decomposed. The EV problem partition for a given demand $q \in Q^{e v}$, which we refer to as $M W_{q}^{e v}(\phi, \sigma \mid \hat{x}, \bar{x})$ is formulated as:

$$
\text { minimize } \sum_{\substack{a \in A_{e v}^{q} \\ i \in K_{a}^{q}}} \bar{x}_{i} \phi_{a}^{q}+\sigma_{q}
$$


subject to

$$
\begin{aligned}
& \sum_{\substack{a \in A_{e v}^{q} \\
i \in K_{a}^{q}}} \hat{x}_{i} \phi_{a}^{q}+\sigma_{q}=\mathbf{o p t}\left(S P D_{q}^{e v}(\phi, \sigma \mid \hat{x})\right) \\
& \sum_{a \in A_{e v}^{q}} \phi_{a}^{q}+\sigma_{q} \geq f_{e v}^{q} d^{q} \\
& \phi_{a}^{q}, \sigma_{q} \geq 0 \quad \forall a \in A_{e v}^{q}
\end{aligned}
$$

Observe that the constraint set enforces the solution to be optimal for the $\operatorname{SPD}_{q}^{e v}(\phi, \sigma \mid \hat{x})$ problem for which we have the optimal solutions presented in Proposition 3. Therefore we can construct the optimal variable values of the $\operatorname{MW}_{q}^{e v}(\phi, \sigma \mid \hat{x}, \bar{x})$ in the following proposition:

Proposition 5. The following solution is optimal for $\operatorname{MW}_{q}^{e v}(\phi, \sigma \mid \hat{x}, \bar{x})$.

$$
\begin{aligned}
& \phi_{a}^{q}= \begin{cases}f_{e v}^{q} d^{q}, & \text { if } z(q)=1,\left|\mathcal{A}_{1}^{q}(\hat{x})\right| \neq 0, a=\operatorname{argmin}_{b \in \mathcal{A}_{1}^{q}(\hat{x})}\left\{\sum_{i \in K_{b}^{q}} \bar{x}_{i}\right\} \text { and } \sum_{i \in K_{a}^{q}} \bar{x}_{i} \leq 1 \\
f_{e v}^{q} d^{q}, & \text { if } z(q)=0 \text { and } a=\operatorname{argmin}_{b \in \mathcal{A}_{0}^{q}(\hat{x})}\left\{\sum_{i \in K_{b}^{q}} \bar{x}_{i}\right\} \\
0, & \text { otherwise }\end{cases} \\
& \sigma_{q}= \begin{cases}f_{e v}^{q} d^{q}, & \text { if } z(q)=1 \text { and } \sum_{i \in K_{a}^{q}} \bar{x}_{i}>1, \forall a \in \mathcal{A}_{1}^{q}(\hat{x}) \\
f_{e v}^{q} d^{q}, & \text { if } z(q)=1 \text { and }\left|\mathcal{A}_{1}^{q}(\hat{x})\right|=0 \\
0, & \text { otherwise }\end{cases}
\end{aligned}
$$

Proof. Let $\mathcal{P}(\phi, \sigma \mid \hat{x})$ be the set of optimal dual variables for $\operatorname{SPD}_{q}^{e v}(\phi, \sigma \mid \hat{x})$ problem as identified in Proposition 3. Then we can remodel the problem without the complicating Constraints (61) as follows:

$$
\text { minimize } \sum_{\substack{a \in A_{e q}^{q} \\ i \in K_{a}^{v}}} \bar{x}_{i} \phi_{a}^{q}+\sigma_{q}
$$

subject to

$$
\left(\sigma_{q}, \phi_{a}^{q}\right) \in \mathcal{P}(\phi, \sigma \mid \hat{x})
$$

Note that we have two conditions in Proposition 3 depending on the optimal value of $z(q)$.

Case (1) If the path $q \in Q$ is not chargeable (i.e., $\left.z_{q}=0\right)$, then the optimal solution of $M W_{q}^{e v}(\phi, \sigma \mid \hat{x}, \bar{x})$ problem is $\phi_{a}^{q}=$ $f_{e v}^{q} d^{q}$ for $a=\operatorname{argmin}_{b \in \mathcal{A}_{0}^{q}(\hat{x})}\left\{\sum_{i \in K_{b}^{q}} \bar{x}_{i}\right\}$ and all of the remaining variables are equal to zero.

Case (2) If a given path $q \in Q^{e v}$ is chargeable (i.e., $\left.z_{q}=1\right)$, then we can find the optimal solution of the $M W_{q}^{e v}(\phi, \sigma \mid \hat{x}, \bar{x})$ problem by investigating the tradeoff between the objective function coefficients of $\sigma$ and $\phi$. First of all, observe that if $\left|\mathcal{A}_{1}^{q}(\hat{x})\right|=0$, then the only optimal solution can be attained by letting $\sigma_{q}=f_{e v}^{q} d^{q}$ and all of the remaining variables equal to zero. Otherwise, if $\left|\mathcal{A}_{1}^{q}(\hat{x})\right| \geq 1$, then we check if $\sum_{i \in K_{a}^{q}} \bar{x}_{i}>1, \forall a \in \mathcal{A}_{1}^{q}(\hat{x})$ in which case, assigning $\sigma=f_{e v}^{q} d^{q}$ and all of the remaining variables equal to zero produces the optimal solution for $M W_{q}^{e v}(\phi, \sigma \mid \hat{x}, \bar{x})$. Lastly, if $\left|\mathcal{A}_{1}^{q}(\hat{x})\right| \neq 0$ and $\sum_{i \in K_{a}^{q}} \bar{x}_{i} \leq 1$, then we select the arc with $a=\operatorname{argmin}_{b \in \mathcal{A}_{1}^{q}(\hat{x})}\left\{\sum_{i \in K_{b}^{q}} \bar{x}_{i}\right\}$ and assign $\phi_{a}^{q}=f_{e v}^{q} d^{q}$ and all of the remaining variables equal to zero. We now have the desired result as presented in Proposition 5.

In a similar fashion, we can construct the optimal variable values of the $M W_{q a}^{\text {phev }}(\mu, \rho \mid \hat{x}, \bar{x})$ problem, which is defined as:

$$
\text { minimize } \sum_{i \in R_{a}^{q} \backslash s(q)} \bar{x}_{i} \mu_{a}^{q i}+\rho_{a}^{q}
$$

subject to

$$
\begin{aligned}
& \sum_{i \in R_{a}^{q} \backslash s(q)} \hat{x}_{i} \mu_{a}^{q i}+\rho_{a}^{q}=\mathbf{o p t}\left(S P D_{q a}^{\text {phev }}(\mu, \rho \mid \hat{x})\right) \\
& \mu_{a}^{q i}+\rho_{a}^{q} \geq f_{\text {phev }}^{q} d_{a}^{q i} \quad \forall i \in R_{a}^{q} \backslash s(q) \\
& \rho_{a}^{q} \geq f_{\text {phev }}^{q} d_{a}^{q s(q)} \quad s(q) \in R_{a}^{q}
\end{aligned}
$$




$$
\mu_{a}^{q i}, \rho_{a}^{q} \geq 0 \quad \forall i \in R_{a}^{q} \backslash s(q)
$$

Proposition 6. The following solution is optimal for $\operatorname{MW}_{q a}^{\text {phev }}(\mu, \rho \mid \hat{x}, \bar{x})$.

(1) If $y_{\hat{a}}^{\hat{q} j}=1$ for $j \neq s(\hat{q})$, then:

$$
\rho_{a}^{q}=\operatorname{argmin}_{\lambda \in S_{a}^{q}}\left(\sum_{\substack{i \in R_{a}^{q} \backslash s(q) \\ i \notin K^{\star}(\hat{x})}}\left(\bar{x}_{i} \max \left\{0, f_{p h e v}^{q} d_{a}^{q i}-\lambda\right\}\right)+\left(1-\bar{x}_{j}\right) \lambda\right)
$$

where $\rho_{\text {max }}=f_{\text {phev }}^{q} d_{a}^{q j}, \rho_{\text {min }}=\max \left\{f_{\text {phev }}^{q} d_{a}^{q s(q)}, \max _{i \in R_{a}^{q} \cap K^{\star}(\hat{x}), i \neq j, i \neq s(q)} f_{\text {phev }}^{q} d_{a}^{q i}\right\}$ and $S_{a}^{q}=\left\{\rho_{\min }, \rho_{\max }\right\} \cup\left\{f_{\text {phev }}^{q} d_{a}^{q i}: \rho_{\text {min }} \leq\right.$ $f_{\text {phev }}^{q} d_{a}^{q i} \leq \rho_{\max }$ and $\left.i \in R_{a}^{q}, \forall i \notin K^{\star}(\hat{x}), i \neq s(q), i \neq j\right\}$

else if $y_{\hat{a}}^{\hat{q} s(\hat{q})}=1$ then, $\rho_{a}^{q}=f_{\text {phev }}^{q} d_{a}^{q s(q)}$

else $\rho_{a}^{q}=0$.

(2) $\mu_{a}^{q i}=\max \left\{0, f_{\text {phev }}^{q} d_{a}^{q i}-\rho_{a}^{q}\right\}, \quad i \in R_{a}^{q} \backslash s(q)$

Proof. Similar to the $M W_{q}^{e v}(\phi, \sigma \mid \hat{x}, \bar{x})$ problem, $M W_{q a}^{\text {phev }}(\mu, \rho \mid \hat{x}, \bar{x})$ can also be modeled using the conditions in Proposition 4 . Note that we have three conditions in Proposition 4 depending on the optimal value of $y_{a}^{q i}$. There are unique optimal solutions for both Case (1) and Case (2). They are also optimal for the $M W_{q a}^{p h e v}(\mu, \rho \mid \hat{x}, \bar{x})$ problem. When we have $y_{a}^{q j}=1$ for $j$ $\neq s(q)$, that is Case (3), $M W_{q a}^{\text {phev }}(\mu, \rho \mid \hat{x}, \bar{x})$ can be written as:

$$
\text { minimize } \sum_{i \in R_{a}^{q} \backslash s(q)} \bar{x}_{i} \mu_{a}^{q i}+\rho_{a}^{q}
$$

subject to

$$
\begin{aligned}
& \mu_{a}^{q j}+\rho_{a}^{q}=f_{\text {phev }}^{q} d_{a}^{q j} \\
& \mu_{a}^{q i}=0 \quad \forall i \in R_{a}^{q}, \forall i \in K^{\star}(\hat{x}), i \neq s(q), i \neq j \\
& \rho_{a}^{q} \geq f_{\text {phev }}^{q} d_{a}^{q i} \quad \forall i \in R_{a}^{q}, \forall i \in K^{\star}(\hat{x}), i \neq s(q), i \neq j \\
& \mu_{a}^{q i}+\rho_{a}^{q} \geq f_{\text {phev }}^{q} d_{a}^{q i} \quad \forall i \in R_{a}^{q}, \forall i \notin K^{\star}(\hat{x}), i \neq s(q), i \neq j \\
& \rho_{a}^{q} \geq f_{\text {phev }}^{q} d_{a}^{q s(q)} \quad \text { if } s(q) \in R_{a}^{q} \\
& \mu_{a}^{q i}, \rho_{a}^{q} \geq 0 \quad \forall i \in R_{a}^{q}, i \notin K^{\star}(\hat{x}), i \neq s(q)
\end{aligned}
$$

Note that the optimality conditions given in Proposition 4 are simply appended as constraints to the above model. This ensures that our optimal solution for $\operatorname{MW}_{q a}^{\text {phev }}(\mu, \rho \mid \hat{x}, \bar{x})$ is also optimal for $\operatorname{SPD}^{\text {phev }}(\mu, \rho \mid \hat{x})$. In this model, we have $\mu_{a}^{q j}=f_{\text {phev }}^{q} d_{a}^{q j}-\rho_{a}^{q}$ due to Constraints (74). Rewriting $\mu_{a}^{q j}$, and letting $\mu_{a}^{q i}=0, \forall i \in R_{a}^{q}, \forall i \in K^{\star}(\hat{x}), i \neq s(q), i \neq j$, we have an equivalent model:

$$
\bar{x}_{j} f_{\text {phev }}^{q} d_{a}^{q j}+\operatorname{minimize} \sum_{\substack{i \in R_{a}^{q} \backslash s(q) \\ i \notin K^{\star}(\hat{x})}} \bar{x}_{i} \mu_{a}^{q i}+\left(1-\bar{x}_{j}\right) \rho_{a}^{q}
$$

subject to

$$
\begin{aligned}
& \rho_{a}^{q} \geq f_{\text {phev }}^{q} d_{a}^{q i} \quad \forall i \in R_{a}^{q}, i \in K^{\star}(\hat{x}), i \neq s(q), i \neq j \\
& \mu_{a}^{q i}+\rho_{a}^{q} \geq f_{\text {phev }}^{q} d_{a}^{q i} \quad \forall i \in R_{a}^{q}, \forall i \notin K^{\star}(\hat{x}), i \neq s(q), i \neq j \\
& \rho_{a}^{q} \geq f_{\text {phev }}^{q} d_{a}^{q s(q)} \quad \text { if } s(q) \in R_{a}^{q} \\
& f_{\text {phev }}^{q} d_{a}^{q j}-\rho_{a}^{q} \geq 0 \\
& \mu_{a}^{q i}, \rho_{a}^{q} \geq 0 \quad \forall i \in R_{a}^{q}, i \notin K^{\star}(\hat{x}), i \neq s(q)
\end{aligned}
$$


Table 1

Road network features.

\begin{tabular}{|c|c|c|c|c|c|c|}
\hline \multirow[t]{2}{*}{ Network } & \multirow[t]{2}{*}{ \#nodes } & \multirow[t]{2}{*}{ \# OD pair nodes } & \multirow[t]{2}{*}{ \#edges } & \multicolumn{3}{|c|}{ node degree } \\
\hline & & & & Min & Max & Mean \\
\hline 25-node & 25 & 300 & 86 & 1 & 6 & 3.36 \\
\hline California (CA) & 339 & 1167 & 1234 & 1 & 7 & 3.64 \\
\hline
\end{tabular}

Observe that Constraints (81), (83) and (84) can be combined as $\rho_{\max } \geq \rho_{a}^{q} \geq \rho_{\min }$, where $\rho_{\max }=f_{\text {phev }}^{q} d_{a}^{q j}$ and $\rho_{\min }=$ $\max \left\{f_{\text {phev }}^{q} d_{a}^{q s(q)}, \max _{i \in R_{a}^{q} \cap K^{*}(\hat{x}), i \neq j, i \neq s(q)} f_{\text {phev }}^{q} d_{a}^{q i}\right\}$. The model is then:

$\bar{x}_{j} f_{\text {phev }}^{q} d_{a}^{q j}+$ minimize $\sum_{\substack{i \in R_{a}^{q} \backslash s(q) \\ i \notin K^{*}(\bar{x})}} \bar{x}_{i} \mu_{a}^{q i}+\left(1-\bar{x}_{j}\right) \rho_{a}^{q}$

subject to

$$
\begin{aligned}
& \mu_{a}^{q i}+\rho_{a}^{q} \geq f_{\text {phev }}^{q} d_{a}^{q i} \quad \forall i \in R_{a}^{q}, \forall i \notin K^{\star}(\hat{x}), i \neq s(q), i \neq j \\
& \rho_{\text {max }} \geq \rho_{a}^{q} \geq \rho_{\text {min }} \\
& \mu_{a}^{q i} \geq 0 \quad \forall i \in R_{a}^{q}, i \notin K^{\star}(\hat{x}), i \neq s(q)
\end{aligned}
$$

In this model, we can determine the optimal $\mu$ values once we know the optimal value of $\rho$. At this point, note that the set of values that $\rho$ can assume are from the set $S_{a}^{q}=\left\{\rho_{\min }, \rho_{\max }\right\} \cup\left\{f_{\text {phev }}^{q} d_{a}^{q i}: \rho_{\min } \leq f_{\text {phev }}^{q} d_{a}^{q i} \leq \rho_{\max }\right.$ and $i \in R_{a}^{q}, \forall i \notin$ $\left.K^{\star}(\hat{x}), i \neq s(q), i \neq j\right\}$. Any other possible value for the $\rho$ variable would either be suboptimal or infeasible. Thus, the following solution is optimal for $M W_{q a}^{\text {phev }}(\mu, \rho \mid \hat{x}, \bar{x})$.

$$
\begin{aligned}
& \rho_{a}^{q}=\operatorname{argmin}_{\lambda \in S_{a}^{q}}\left(\sum_{\substack{i \in R_{a}^{q} \backslash s(q) \\
i \notin K^{\star}(\bar{x})}}\left(\bar{x}_{i} \max \left\{0, f_{\text {phev }}^{q} d_{a}^{q i}-\lambda\right\}\right)+\left(1-\bar{x}_{j}\right) \lambda\right) \\
& \mu_{a}^{q i}=\max \left\{0, f_{\text {phev }}^{q} d_{a}^{q i}-\rho_{a}^{q}\right\}
\end{aligned}
$$

This completes the proof.

Remark 3. In a regular MW framework, two different LP problems need to be solved for every subproblem, one to obtain the optimal value of the subproblem, and one for the MW problem. However, in our particular case, we are able to construct the primal subproblem solution, the dual subproblem solution and a Pareto-optimal cut directly, without any need to build an LP.

\section{Computational study}

In this section, we present performance analyses of the CSLM-PHEV model and the BD algorithm with three different cut selection schemes. Note that in all three decomposition implementations, we only construct one search tree, and solve the master problem using branch-and-cut method. In order to compare the computational performances of the solution techniques, computational studies are carried out on two datasets. The first one, shown in Fig. 2, is a 25-node dataset commonly used in the literature (Hodgson, 1990; Kuby and Lim, 2005; MirHassani and Ebrazi, 2013). A second dataset is a real-world representation of the California (CA) road network as presented in Fig. 3 (Arslan et al., 2015; Ylldı et al., 2015). The data related to network specifications are presented in Table 1.

All of the instances are run on a desktop computer with $3.16 \mathrm{GHz} 2 \times \mathrm{xDuo}$ CPU, 2.00GB RAM. The algorithms are implemented using Java and CPLEX 12.5 (IBM, 2013) with Concert Technology.

\subsection{5-Node road network}

In the 25-node road network, all nodes are assumed to be origin and destination nodes similar to previous studies. Following the convention in the literature, we assume that the demand between OD pairs is directly proportional with the populations of the origin and the destination nodes, and inversely proportional with the distance between the OD pair, and the calculation of the vehicle flow is due to gravity model by Hodgson (1990). The ranges considered in the 


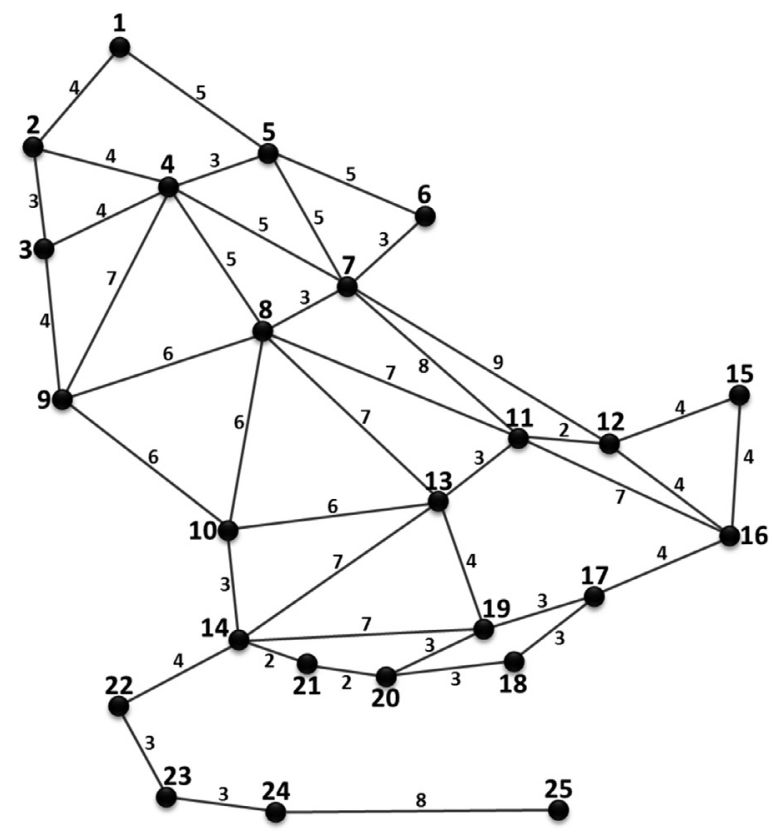

Fig. 2. 25-node road network.

Table 2

25-node road network results.

\begin{tabular}{|c|c|c|c|c|c|c|c|c|c|c|c|c|c|c|c|c|c|c|c|c|c|c|c|}
\hline \multicolumn{2}{|c|}{ Problem } & \multirow[b]{2}{*}{ 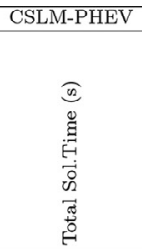 } & \multicolumn{7}{|c|}{ Singlecut Implementation } & \multicolumn{7}{|c|}{ Multicut Implementation } & \multicolumn{7}{|c|}{ Pareto-optimal Cut Implementation } \\
\hline $\mathrm{p}$ & 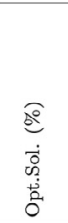 & & 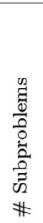 & $\begin{array}{l}\stackrel{\infty}{5} \\
己 \\
\#\end{array}$ & 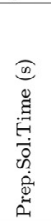 & 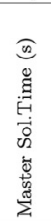 & 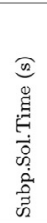 & 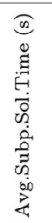 & 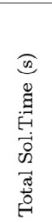 & 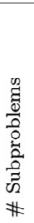 & 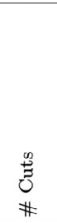 & 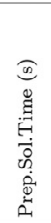 & 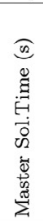 & 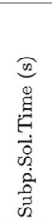 & 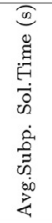 & 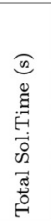 & 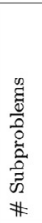 & $\begin{array}{l}\stackrel{n}{E} \\
\text { E } \\
\#\end{array}$ & 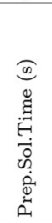 & 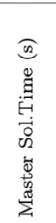 & 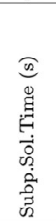 & 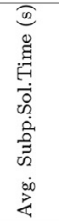 & 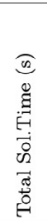 \\
\hline 1 & 23.07 & 1.35 & 3 & 2 & 0.03 & 0.07 & 0.09 & 0.03 & 0.18 & 2 & 4212 & 0.03 & 0.08 & 0.27 & 0.13 & 0.37 & 3 & 2 & 0.05 & 0.12 & 0.11 & 0.04 & 0.28 \\
\hline 2 & 32.60 & 0.60 & 10 & 9 & 0.04 & 0.01 & 0.13 & 0.01 & 0.19 & 4 & 4638 & 0.08 & 0.14 & 0.44 & 0.11 & 0.65 & $y$ & 7 & 0.05 & 0.19 & 0.29 & 0.03 & 0.52 \\
\hline 3 & 40.76 & 0.89 & 34 & 30 & 0.02 & 0.02 & 0.44 & 0.01 & 0.48 & 6 & 4948 & 0.04 & 0.19 & 0.60 & 0.10 & 0.83 & 15 & 11 & 0.05 & 0.18 & 0.37 & 0.02 & 0.60 \\
\hline 4 & 50.55 & 0.53 & 42 & 37 & 0.02 & 0.04 & 0.53 & 0.01 & 0.58 & 4 & 4862 & 0.03 & 0.17 & 0.43 & 0.11 & 0.63 & 16 & 13 & 0.05 & 0.18 & 0.41 & 0.03 & 0.64 \\
\hline 5 & 59.17 & 0.64 & 51 & 47 & 0.02 & 0.05 & 0.65 & 0.01 & 0.72 & 4 & 4947 & 0.04 & 0.15 & 0.45 & 0.11 & 0.65 & 15 & 11 & 0.05 & 0.18 & 0.40 & 0.03 & 0.62 \\
\hline 6 & 66.38 & 0.50 & 56 & 52 & 0.02 & 0.07 & 0.71 & 0.01 & 0.79 & 4 & 5071 & 0.03 & 0.19 & 0.48 & 0.12 & 0.71 & 11 & 9 & 0.05 & 1.45 & 0.30 & 0.03 & 1.81 \\
\hline 7 & 71.29 & 0.47 & 85 & 80 & 0.02 & 0.12 & 1.07 & 0.01 & 1.20 & 5 & 5188 & 0.03 & 0.15 & 0.70 & 0.14 & 0.88 & 17 & 14 & 0.05 & 0.18 & 0.46 & 0.03 & 0.69 \\
\hline 8 & 75.88 & 0.41 & 125 & 118 & 0.02 & 0.18 & 1.56 & 0.01 & 1.76 & 7 & 5192 & 0.03 & 0.15 & 0.82 & 0.12 & 1.01 & 15 & 13 & 0.05 & 0.17 & 0.42 & 0.03 & 0.65 \\
\hline 9 & 80.17 & 0.38 & 138 & 132 & 0.02 & 0.20 & 1.72 & 0.01 & 1.9 & 6 & 5246 & 0.03 & 0.15 & 0.77 & 0.13 & 0.96 & 13 & 9 & 0.05 & 0.17 & 0.37 & 0.03 & 0.59 \\
\hline 10 & 84.75 & 0.32 & 105 & 99 & 0.02 & 0.15 & 1.42 & 0.01 & 1.58 & 5 & 5249 & 0.03 & 0.11 & 0.70 & 0.14 & 0.84 & 9 & 6 & 0.05 & 0.15 & 0.27 & 0.03 & 0.47 \\
\hline 11 & 87.55 & 0.31 & 103 & 95 & 0.02 & 0.16 & 1.28 & 0.01 & 1.46 & 9 & 5420 & 0.03 & 0.13 & 1.14 & 0.13 & 1.30 & 18 & 12 & 0.05 & 0.20 & 0.51 & 0.03 & 0.76 \\
\hline 12 & 90.16 & 31 & 112 & 102 & 0.02 & 0.15 & 1.39 & 0.01 & 1.56 & 7 & 5555 & 0.03 & 0.13 & 0.91 & 0.13 & 1.08 & 13 & 11 & 0.05 & 0.17 & 0.40 & 0.03 & 0.62 \\
\hline 13 & 92.78 & 5 & 95 & 89 & 0.02 & 0.11 & 1.17 & 0.0 & 1.3 & 7 & 5578 & 0.03 & 0.1 & 0.86 & 0.12 & 1.03 & 16 & 12 & 0.05 & 0.18 & 0.43 & 0.03 & 0.66 \\
\hline 14 & 95.11 & 0.29 & 63 & 58 & 0.02 & 0.06 & 0.79 & 0.01 & 0.87 & 6 & 5584 & 0.03 & 0.11 & 0.78 & 0.13 & 0.92 & 12 & 9 & 0.05 & 0.17 & 0.38 & 0.03 & 0.60 \\
\hline 15 & 97.12 & 0.27 & 45 & 35 & 0.02 & 0.03 & 0.56 & 0.01 & 0.61 & 6 & 5632 & 0.03 & 0.11 & 0.79 & 0.13 & 0.93 & 9 & 8 & 0.05 & 0.15 & 0.28 & 0.03 & 0.48 \\
\hline 16 & 98.69 & 0.26 & 27 & 21 & 0.02 & 0.02 & 0.34 & 0.01 & 0.38 & 6 & 5627 & 0.03 & 0.12 & 0.78 & 0.13 & 0.94 & 7 & 6 & 0.05 & 0.15 & 0.25 & 0.04 & 0.45 \\
\hline 17 & 99.58 & 0.22 & 26 & 21 & 0.02 & 0.02 & 0.32 & 0.0 & 0.36 & 4 & 5657 & 0.03 & 0.0 & 0.52 & 0.13 & 0.62 & 8 & 6 & 0.05 & 0.17 & 0.30 & 0.04 & 0.52 \\
\hline 18 & 99.86 & 29 & 22 & 17 & 0.02 & 0.02 & 0.28 & 0.0 & 0.31 & 4 & 5683 & 0.07 & 0.06 & 0.53 & 0.13 & 0.66 & 4 & 3 & 0.05 & 0.15 & 0.18 & 0.04 & 0.37 \\
\hline 19 & 100.00 & 0.22 & 6 & 4 & 0.02 & 0.01 & 0.08 & 0.01 & 0.11 & 4 & 5730 & 0.03 & 0.05 & 0.50 & 0.12 & 0.59 & 4 & 3 & 0.05 & 0.15 & 0.18 & 0.04 & 0.37 \\
\hline 20 & 100.00 & 0.22 & 3 & 2 & 0.02 & 0.01 & 0.04 & 0.01 & 0.07 & 3 & 5709 & 0.03 & 0.05 & 0.42 & 0.14 & 0.50 & 3 & 2 & 0.05 & 0.14 & 0.13 & 0.04 & 0.32 \\
\hline 21 & 100.00 & 0.22 & 3 & 2 & 0.02 & 0.01 & 0.04 & 0.01 & 0.07 & 3 & 5709 & 0.03 & 0.04 & 0.51 & 0.17 & 0.59 & 3 & 2 & 0.05 & 0.14 & 0.13 & 0.04 & 0.32 \\
\hline 22 & 100.00 & & 3 & 2 & 0.02 & 0.01 & 0.04 & 0.0 & $0 .($ & 3 & 5709 & 0.03 & 0.05 & 0.44 & 0.15 & 0.5 & 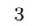 & 2 & 0.05 & 0.14 & 0.13 & 0.04 & 0.32 \\
\hline 23 & 100.00 & 0.22 & 3 & 2 & 0.02 & 0.01 & 0.04 & 0.01 & 0.06 & 3 & 5709 & 0.03 & 0.05 & 0.39 & 0.13 & 0.47 & 3 & 2 & 0.05 & 0.14 & 0.13 & 0.04 & 0.32 \\
\hline 24 & 100.00 & 0.30 & 3 & 2 & 0.02 & 0.01 & 0.05 & 0.02 & 0.07 & 3 & 5709 & 0.04 & 0.06 & 0.41 & 0.14 & 0.51 & 3 & 2 & 0.05 & 0.14 & 0.13 & 0.04 & 0.32 \\
\hline 25 & 100.00 & 0.12 & 2 & 1 & 0.02 & 0.01 & 0.03 & 0.01 & 0.05 & 2 & 2719 & 0.03 & 0.02 & 0.27 & 0.13 & 0.32 & 2 & 1 & 0.05 & 0.14 & 0.11 & 0.05 & 0.30 \\
\hline
\end{tabular}

25-node network in previous studies are 4, 8, and 12. Since we can handle multiple vehicle types in the same problem, we consider EVs and PHEVs with ranges 4, 8 and 12 for every OD pair with equal flows as attained from the gravity model. With these settings, we solved the problem using the BD algorithm with singlecut, multicut and Pareto-optimal cut generation schemes. The $p$ value range is from 1 to 25 . The results are presented in Table 2 . In the table, the second column represents the optimal solution value. We express this value as the percentage of covered distance with respect to the total coverable distance. Total coverable distance is defined as the difference in the objective function value of the CSLM-PHEV model when a charging station is located at all of the nodes in the network and when no charging station is located in the network. The third column is the solution time of the CSLM-PHEV model. Corresponding to each of the three 


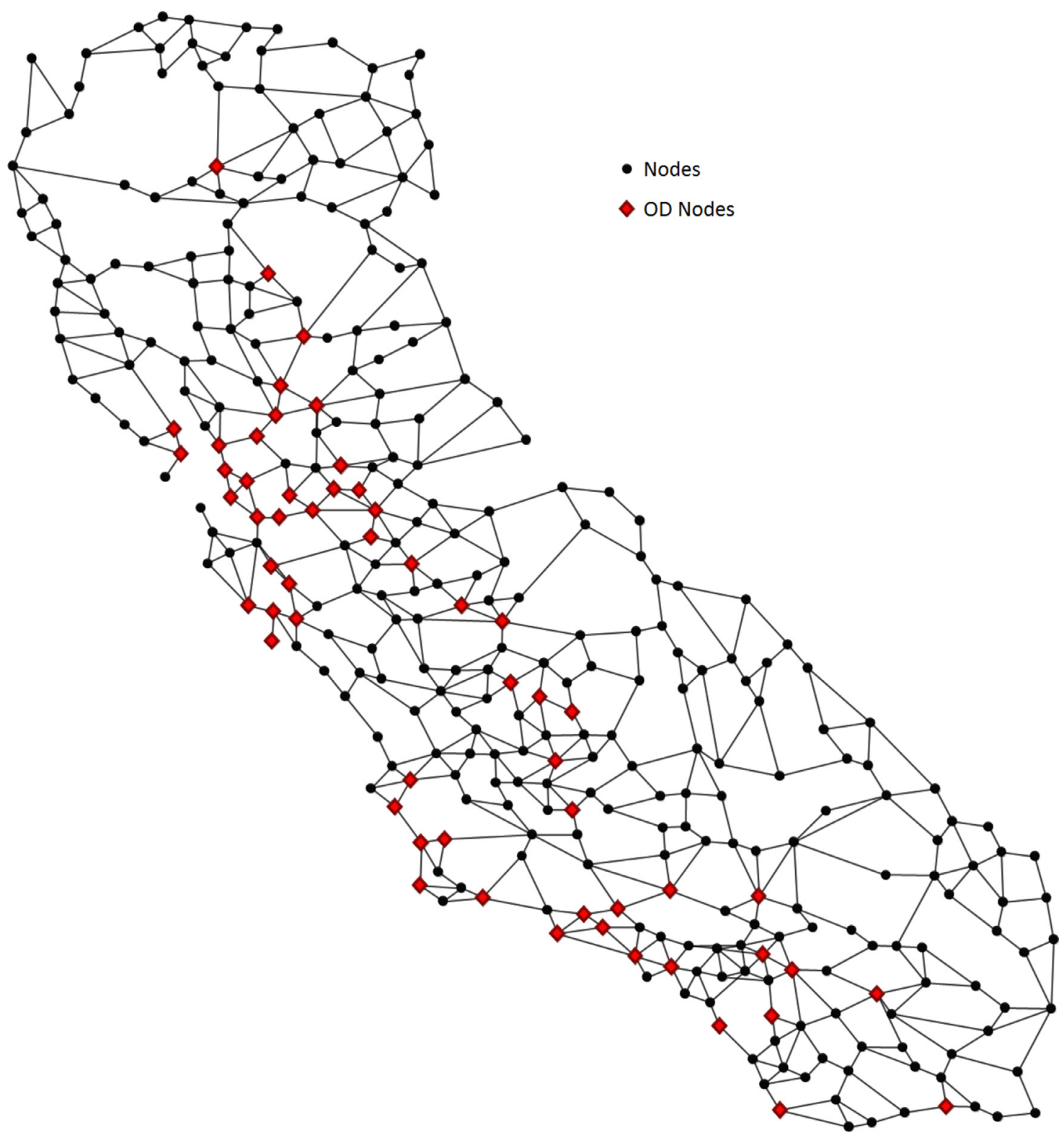

Fig. 3. California state road network.

BD implementations, there are seven columns. \# Subproblems shows the number of subproblems solved, \# Cuts is the total number of cuts added, Prep.Sol.Time, Master Sol.Time and Subp.Sol.Time show the preprocessing time, master problem solution time and the subproblem solution time, respectively. Avg.Subp.Sol.Time is the average solution time of a single subproblem, found by dividing the Subp.Sol.Time by the \# Subproblems. Total Sol.Time column is the total running time, which equals to the summation of preprocessing, subproblem and master problem solution times.

There are some critical insights regarding the nature of the solution techniques. The BD algorithm with singlecut implementation generally solves the most number of subproblems while the multicut implementations requires to solve the least number of subproblems to arrive at the optimal solution. However, the number of cuts added to the master problem is far more in multicut implementation than the other two cut implementations. The Pareto-optimal cut implementation solves more subproblems than the multicut, but the cuts added are far less in number. On the average, the Pareto-optimal cut generation scheme solves the problems faster than the other two cut generation techniques. As it will be apparent in the following parts, similar line of results can be observed in larger sized networks. 
Table 3

EV sales.

\begin{tabular}{lll}
\hline Designator & Range $(\mathrm{km})$ & Percentage in total EV sales (\%) \\
\hline EV-1 & 426 & 25.43 \\
EV-2 & 142 & 19.58 \\
EV-3 & 132 & 54.99 \\
\hline
\end{tabular}

Table 4

PHEV sales.

\begin{tabular}{lll}
\hline Designator & Range $(\mathrm{km})$ & Percentage in total PHEV sales (\%) \\
\hline PHEV-1 & 61 & 45.13 \\
PHEV-2 & 32 & 20.46 \\
PHEV-3 & 19 & 34.41 \\
\hline
\end{tabular}

Table 5

California road network results.

\begin{tabular}{|c|c|c|c|c|c|c|c|c|c|c|c|c|c|c|c|c|c|c|}
\hline \multicolumn{2}{|c|}{ Problem } & \multirow[b]{2}{*}{ 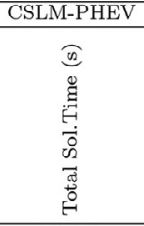 } & \multicolumn{6}{|c|}{ Singlecut Implementation } & \multicolumn{5}{|c|}{ Multicut Implementation } & \multicolumn{5}{|c|}{ Pareto-optimal Cut Implementation } \\
\hline $\mathrm{p}$ & 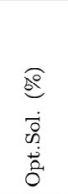 & & 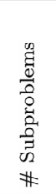 & $\begin{array}{l}\text { 暏 } \\
\text { \# } \\
\text { \# }\end{array}$ & 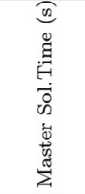 & 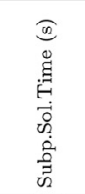 & 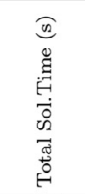 & 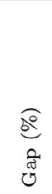 & 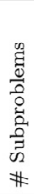 & $\begin{array}{l}\stackrel{\Xi}{\exists} \\
\text { U }\end{array}$ & 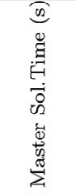 & 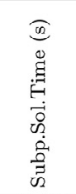 & 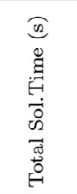 & 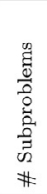 & 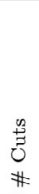 & 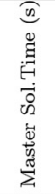 & 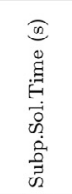 & 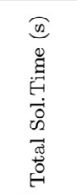 \\
\hline 1 & 40.84 & 1438.72 & 6 & 5 & 0.26 & 4.07 & 7.86 & - & 2 & 58665 & 10.33 & 31.70 & 48.24 & 3 & 2 & 0.14 & 2.49 & 6.10 \\
\hline 2 & 59.04 & 1258.87 & 20 & 14 & 0.03 & 13.45 & 16.42 & - & 2 & 58665 & 4.19 & 31.28 & 40.87 & 8 & 5 & 0.14 & 6.85 & 10.46 \\
\hline 3 & 66.38 & 1335.37 & 53 & 46 & 0.25 & 36.91 & 40.10 & - & 4 & 66563 & 11.37 & 98.20 & 114.96 & 12 & 8 & 0.13 & 11.02 & 14.61 \\
\hline 4 & 70.27 & 1547.52 & 215 & 209 & 1.20 & 150.74 & 154.89 & - & 8 & 73896 & 29.77 & 172.13 & 207.32 & 18 & 11 & 0.15 & 17.49 & 21.11 \\
\hline 5 & 73.64 & 1626.40 & 572 & 559 & 9.36 & 413.04 & 425.44 & - & 7 & 75363 & 33.96 & 155.03 & 194.42 & 24 & 17 & 0.16 & 24.90 & 28.53 \\
\hline 6 & 76.92 & 1352.06 & 792 & 782 & 25.70 & 561.75 & 590.39 & - & 5 & 78716 & 29.85 & 113.36 & 148.65 & 20 & 15 & 0.15 & 20.46 & 24.09 \\
\hline 7 & 79.47 & 1850.89 & 1397 & 1384 & 128.41 & 996.71 & 1128.11 & - & 7 & 85985 & 32.07 & 179.31 & 216.60 & 28 & 19 & 0.16 & 30.10 & 33.75 \\
\hline 8 & 82.02 & 1576.26 & 1247 & 1238 & 198.84 & 882.96 & 1084.82 & - & 6 & 88099 & 27.50 & 166.40 & 199.18 & 35 & 23 & 0.20 & 39.17 & 42.85 \\
\hline 9 & 84.27 & 1128.04 & 1023 & 1009 & 162.98 & 734.19 & 900.09 & - & 8 & 85900 & 30.61 & 220.09 & 256.28 & 22 & 16 & 0.18 & 27.54 & 31.20 \\
\hline 10 & 86.02 & 1136.14 & 991 & 975 & 208.00 & 726.91 & 937.92 & - & 5 & 88709 & 36.31 & 133.95 & 175.97 & 28 & 21 & 0.23 & 31.35 & 35.05 \\
\hline 11 & 87.58 & 1318.24 & 850 & 836 & 222.13 & 622.46 & 847.60 & - & 5 & 88912 & 41.66 & 132.07 & 179.93 & 24 & 17 & 0.23 & 26.51 & 30.22 \\
\hline 12 & 89.10 & 880.49 & 463 & 447 & 157.83 & 330.18 & 490.99 & - & 5 & 88577 & 20.76 & 131.76 & 158.70 & 30 & 20 & 0.26 & 34.73 & 38.47 \\
\hline 13 & 90.30 & 879.33 & 373 & 354 & 79.50 & 272.73 & 355.14 & - & 6 & 90106 & 27.84 & 179.12 & 213.63 & 30 & 20 & 0.22 & 33.64 & 37.32 \\
\hline 14 & 91.28 & 656.85 & 318 & 303 & 136.29 & 226.66 & 365.88 & - & 5 & 89886 & 31.81 & 155.67 & 194.13 & 29 & 19 & 0.23 & 32.76 & 36.45 \\
\hline 15 & 91.94 & 631.22 & 662 & 650 & 915.42 & 485.42 & 1403.86 & - & 4 & 89693 & 52.89 & 133.40 & 193.04 & 33 & 24 & 0.23 & 37.99 & 41.69 \\
\hline 16 & 92.53 & 674.54 & 1384 & 1373 & 2615.08 & 984.84 & 3602.89 & 0.19 & 6 & 90455 & 41.31 & 184.44 & 232.62 & 36 & 26 & 0.29 & 41.61 & 45.37 \\
\hline 17 & 93.07 & 648.92 & 2267 & 2246 & 1985.06 & 1614.86 & 3602.88 & 0.29 & 6 & 90523 & 38.41 & 208.56 & 254.84 & 46 & 34 & 0.26 & 54.42 & 58.14 \\
\hline 18 & 93.58 & 640.30 & 2008 & 1989 & 2135.91 & 1464.04 & 3602.98 & 0.38 & 9 & 93020 & 36.89 & 306.72 & 351.38 & 48 & 34 & 0.44 & 56.84 & 60.75 \\
\hline 19 & 94.05 & 569.41 & 2912 & 2897 & 1487.57 & 2112.40 & 3602.92 & 0.44 & 8 & 93526 & 34.42 & 314.92 & 357.77 & 54 & 39 & 0.42 & 64.21 & 68.10 \\
\hline 20 & 94.49 & 643.31 & 2692 & 2671 & 1684.34 & 1915.58 & 3602.86 & 0.41 & 7 & 93856 & 46.54 & 297.96 & 353.66 & 52 & 42 & 0.55 & 62.95 & 66.99 \\
\hline 30 & 97.19 & 643.48 & 3289 & 3260 & 1239.46 & 2360.48 & 3602.97 & 0.51 & 15 & 101608 & 75.82 & 314.60 & 396.63 & 94 & 73 & 1.07 & 117.83 & 122.38 \\
\hline 40 & 98.57 & 508.04 & 3278 & 3247 & 1266.05 & 2334.53 & 3603.56 & 0.32 & 15 & 105093 & 200.75 & 315.07 & 521.23 & 90 & 74 & 3.66 & 115.79 & 122.91 \\
\hline 50 & 99.31 & 378.55 & 3177 & 3147 & 1357.27 & 2242.65 & 3602.89 & 0.19 & 12 & 106324 & 178.92 & 284.71 & 469.03 & 102 & 80 & 2.32 & 131.88 & 137.67 \\
\hline 60 & 99.74 & 409.56 & 1419 & 1383 & 2608.32 & 991.69 & 3602.96 & 0.06 & 20 & 106796 & 242.35 & 458.60 & 706.33 & 110 & 88 & 5.79 & 142.47 & 151.72 \\
\hline 70 & 99.93 & 237.89 & 291 & 258 & 3400.67 & 200.70 & 3604.88 & 0.01 & 10 & 106910 & 120.71 & 229.34 & 355.45 & 81 & 61 & 0.67 & 103.26 & 107.40 \\
\hline 80 & 99.97 & 179.91 & 185 & 158 & 3.15 & 126.46 & 132.59 & - & 7 & 107154 & 74.11 & 191.22 & 270.73 & 77 & 55 & 0.60 & 98.59 & 102.66 \\
\hline 90 & 99.98 & 123.81 & 146 & 124 & 0.81 & 98.14 & 101.90 & - & 7 & 106825 & 41.91 & 181.78 & 229.04 & 53 & 38 & 0.40 & 67.15 & 71.02 \\
\hline 100 & 100.00 & 208.42 & 130 & 112 & 0.71 & 84.89 & 89.11 & - & 4 & 106568 & 27.03 & 119.42 & 151.66 & 44 & 37 & 0.25 & 55.92 & 59.63 \\
\hline
\end{tabular}

\subsection{California road network}

The CA dataset is a real world representation of the California road network with 339 nodes and 1234 arcs. 57 centers with a population of 50,000 or above are selected as the origin and destination nodes. Similar to Yildiz et al. (2015), we consider all pairings of these nodes with at least 30 miles apart. There are 1167 such pairings as presented in Table 1 . As done in the 25-node network case, the vehicle flows between pairings are calculated using the gravity model (Hodgson, 1990).

Related to EV and PHEV sales numbers, Center for Sustainable Energy (2015) reports new-vehicle rebates on behalf of the California Air Resource Boards Clean Vehicle Rebate Project (CVRP). We can extract EV and PHEV sales versus brands to get the sales percentages in California State for vehicles with different ranges. Table 3 shows that 25.43\% of the total EV sales have an extended range of $426 \mathrm{~km}$ (265 miles). Similarly, Table 4 shows that $45.13 \%$ of the PHEVs have a range of $61 \mathrm{~km}$ (38 miles). Therefore, we have 6 copies for each of the 1167 OD pairs, three of them representing the EVs with different ranges, and the remaining three representing the PHEVs with different ranges.

The California results are presented in Table 5 for problem instances ranging from $p=1$, through $p=20$ with increments of one. After 20 charging stations where the coverage reaches to $94.49 \%$, we increased $p$ by 10 , through 100 , where the coverage reaches to $100.00 \%$. Optimal solutions could be obtained by the CSLM-PHEV model and the BD algorithm with multicut and Pareto-optimal cut implementations. However singlecut implementation terminated with an optimality gap of 
Table 6

Summary of computational results.

\begin{tabular}{llrrr}
\hline Parameter & CSLM-PHEV & Singlecut & Multicut & Pareto-optimal \\
\hline Avg. \# Subproblems & - & 1148.57 & 7.32 & 43.96 \\
Avg. \# Cuts & - & 1131.29 & 89871.18 & 32.79 \\
Avg. prep. sol.time (s) & - & 3.03 & 6.12 & 3.47 \\
Avg. master sol.time (s) & - & 786.81 & 56.43 & 0.70 \\
Avg. subp. sol.time (s) & - & 821.05 & 194.31 & 53.21 \\
Avg. subp. sol. time (s) & - & 0.71 & 26.91 & 1.14 \\
Avg. total sol. time (s) & 874.38 & $>1610.88$ & 256.87 & 57.38 \\
\hline
\end{tabular}

less than $0.51 \%$ for $p=16$ to $p=70$ within an hour time-limit. The multicut implementation solution time is much smaller than singlecut implementation, however, it performs worse than the CSLM-PHEV model for instances with $p=50$ to $p=90$. Also note that the number of cuts added are, on the average, 89871.18 (Table 6). The results indicate that the number of subproblems and the solution times of the Pareto-optimal cut implementation do not get overwhelmed for increasing values of $p$. As seen in Table 6, the average number of subproblems is 43.96 with an average solution time of less than one minute, which is on the average 15 times faster than the CSLM-PHEV model. Our BD algorithm with Pareto-optimal cut construction performs much faster than the CSLM-PHEV model and the other two cut construction techniques especially for smaller $p$ values. The average speedup is nearly 30 times for $p \leq 20$. For greater $p$ values, the runtime of the CSLM-PHEV model also decreases and gets closer to the runtimes of the Pareto-optimal cut implementation of the BD algorithm. It is worth noting that even though BD algorithm is mostly used with the objective of solving problems of larger sizes rather than efficiency concerns, our implementation not only guarantees larger problem sizes (due to decomposition) but also presents promising results for the solution time reduction as well. To confirm this assertion, we also doubled the EV and PHEV demands for the CA network. That is, we considered 12 copies of demand for each OD pair rather than 6 . In that case, the results of Pareto-optimal cut implementation are presented in Table 7. The CSLM-PHEV model cannot even write the problem into the memory and results in out-of-memory error for every instance. However, BD algorithm does not explicitly create all the variables in the solution and continues to solve these large-scale instances within reasonable times. Duplicating the demand does not change the number of binary variables in the CSLP-PHEV problem, however the increase in the number of variables (namely the $y$ and $z$ variables) is drastic. Thus, the CSLM-PHEV model fails to solve the problem. For the BD algorithm, the subproblem solutions and the cuts are constructed in closed form, and this reduces the requirement for extensive memory.

Table 8 lists the optimal charging station locations for $p=1$ through 20. The first column shows the $p$ value. For a given row, an ' $x$ ' sign shows that the corresponding node, as depicted in Fig. 4, is selected. For example, for $p=4$, nodes 2,3 , 5 and 6 are the optimal locations of the charging stations. Observe that the optimal locations of the charging stations are robust in the sense that a node appearing in one solution shows up again in several different forthcoming solutions with increasing $p$ values. Similar findings have been recognized in the literature for the flow-covering problems (Upchurch et al., 2009).

If a node does not show up again, it is generally substituted by other nodes. For instance, node 1 shows up in most of the optimal solutions for $p=1$ through 9 . Then, nodes 12 and 13 start showing up in the optimal solutions for $p=10$ and onwards. Looking at Fig. 4, we observe that these nodes are geographically right next to each other. The same is also true for different node combinations as well. In particular, Node 1 leaves the optimal solution set and nodes 12 and 13 enter; node 7 leaves and nodes 2 and 4 enter; node 8 leaves and nodes 16 and 17 enter; node 11 leaves and node 14 enters; and node 14 leaves and node 11 reenters the optimal solution set again.

For $p=20$, all nodes shown in Fig. 4 except 1, 7, 8 and 14 are selected as charging station locations. These 4 nodes, as shown above, are substituted by other nodes in the network. Another point regarding the optimal charging station locations is that five of these 20 charging station nodes are also OD pairs. This number is quite significant, given that vehicles start with a full battery.

The initial charging stations start showing up in the middle regions of the state on the highway nodes. Then, the selected nodes start spreading over to north and to the south of the initially selected node. The first seven charging stations gradually cover the highway nodes to connect the northern cities to the southern cities. The eighth charging station node connects Los Angeles to San Diego. Similarly, the ninth selected node connects San Francisco to Sacramento. According to the results, initially connecting the northern region to the southern region is critical.

\subsection{Solving the flow refueling location problem by the Benders decomposition approach}

Observe that the CSLP-PHEV is a generalization of FRLP. Therefore the BD algorithm proposed in earlier sections can also be implemented for FRLM. In this subsection, our objective is to test the performance of the BD approach with Paretooptimal cut implementation (BD-PO) on the solution of FRLP. For this purpose, we consider the California dataset with 1167 OD pairs, and only EVs but no PHEVs. Between each OD pair, we have $t \in\{2,3, \ldots, 9\}$ types of EVs with ranges varying between 50 and $150 \mathrm{~km}$. 
Table 7

California road network results with demand doubled.

\begin{tabular}{|c|c|c|c|c|c|c|c|}
\hline \multicolumn{2}{|c|}{ Problem } & \multicolumn{6}{|c|}{ Pareto-optimal cut implementation } \\
\hline$p$ & $\begin{array}{l}\text { S0 } \\
\dot{0} \\
0 \\
02 \\
0 \\
0 \\
0\end{array}$ & $\begin{array}{l}\text { D } \\
\text { ह्व } \\
0 \\
0 \\
0 \\
0 \\
0 \\
0 \\
0 \\
\text { क } \\
\text { \# }\end{array}$ & 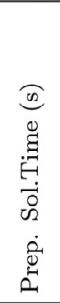 & 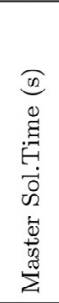 & 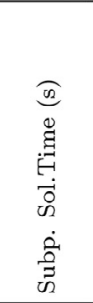 & 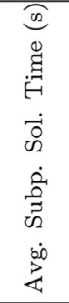 & 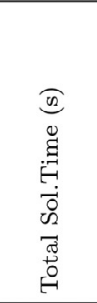 \\
\hline 1 & 40.84 & 3 & 9.85 & 0.14 & 75.95 & 25.32 & 85.94 \\
\hline 2 & 59.04 & 8 & 9.88 & 0.12 & 224.42 & 28.05 & 234.43 \\
\hline 3 & 66.38 & 12 & 9.89 & 0.13 & 360.94 & 30.08 & 370.96 \\
\hline 4 & 70.27 & 18 & 9.88 & 0.15 & 578.17 & 32.12 & 588.19 \\
\hline 5 & 73.64 & 24 & 9.89 & 0.16 & 861.42 & 35.89 & 871.47 \\
\hline 6 & 76.92 & 20 & 9.87 & 0.15 & 713.06 & 35.65 & 723.09 \\
\hline 7 & 79.47 & 28 & 9.90 & 0.16 & 1064.44 & 38.02 & 1074.50 \\
\hline 8 & 82.02 & 35 & 9.89 & 0.20 & 1421.13 & 40.60 & 1431.22 \\
\hline 9 & 84.27 & 22 & 9.90 & 0.17 & 876.52 & 39.84 & 886.60 \\
\hline 10 & 86.02 & 28 & 9.88 & 0.21 & 1138.07 & 40.65 & 1148.15 \\
\hline 11 & 87.58 & 24 & 9.90 & 0.18 & 974.51 & 40.60 & 984.58 \\
\hline 12 & 89.10 & 30 & 9.90 & 0.22 & 1275.83 & 42.53 & 1285.95 \\
\hline 13 & 90.30 & 30 & 9.88 & 0.17 & 1244.35 & 41.48 & 1254.40 \\
\hline 14 & 91.28 & 29 & 9.90 & 0.18 & 1209.51 & 41.71 & 1219.60 \\
\hline 15 & 91.94 & 33 & 9.91 & 0.19 & 1422.25 & 43.10 & 1432.35 \\
\hline 16 & 92.53 & 35 & 9.90 & 0.37 & 1523.78 & 43.54 & 1534.05 \\
\hline 17 & 93.07 & 49 & 9.88 & 0.24 & 2210.21 & 45.11 & 2220.32 \\
\hline 18 & 93.58 & 48 & 9.89 & 0.41 & 2197.71 & 45.79 & 2208.01 \\
\hline 19 & 94.05 & 53 & 9.88 & 0.34 & 2429.80 & 45.85 & 2440.02 \\
\hline 20 & 94.49 & 60 & 9.88 & 0.32 & 2866.34 & 47.77 & 2876.54 \\
\hline 30 & 97.19 & 79 & 9.88 & 1.28 & 4000.15 & 50.63 & 4011.31 \\
\hline 40 & 98.57 & 86 & 9.90 & 3.83 & 4545.76 & 52.86 & 4559.48 \\
\hline 50 & 99.31 & 101 & 9.89 & 6.66 & 5388.40 & 53.35 & 5404.95 \\
\hline 60 & 99.74 & 99 & 9.90 & 7.06 & 5317.02 & 53.71 & 5333.98 \\
\hline 70 & 99.93 & 83 & 9.89 & 0.60 & 4411.93 & 53.16 & 4422.42 \\
\hline 80 & 99.97 & 65 & 9.88 & 0.42 & 3427.71 & 52.73 & 3438.01 \\
\hline 90 & 99.98 & 46 & 9.88 & 0.31 & 2413.95 & 52.48 & 2424.14 \\
\hline 100 & 100.00 & 43 & 9.88 & 0.20 & 2248.53 & 52.29 & 2258.61 \\
\hline
\end{tabular}

Table 8

Optimal charging station locations for California road network.

\begin{tabular}{|c|c|c|c|c|c|c|c|c|c|c|c|c|c|c|c|c|c|c|c|c|c|c|c|c|}
\hline $\mathrm{p}$ & 1 & 2 & 3 & 4 & 5 & 6 & 7 & 8 & 9 & 10 & 11 & 12 & 13 & 14 & 15 & 16 & 17 & 18 & 19 & 20 & 21 & 22 & 23 & 24 \\
\hline 1 & $\times$ & & & & & & & & & & & & & & & & & & & & & & & \\
\hline 2 & & $\times$ & $\times$ & & & & & & & & & & & & & & & & & & & & & \\
\hline 3 & $\times$ & & & $x$ & $x$ & & & & & & & & & & & & & & & & & & & \\
\hline 4 & & $\times$ & $\times$ & & $x$ & $x$ & & & & & & & & & & & & & & & & & & \\
\hline 5 & $\times$ & & $\times$ & & $x$ & $x$ & $x$ & & & & & & & & & & & & & & & & & \\
\hline 6 & $\times$ & & $\times$ & & $x$ & $x$ & $x$ & $x$ & & & & & & & & & & & & & & & & \\
\hline 7 & $\times$ & & $\times$ & & $x$ & $\times$ & $\times$ & $\times$ & $\times$ & & & & & & & & & & & & & & & \\
\hline 8 & $\times$ & & $\times$ & & $x$ & $x$ & $x$ & $x$ & $x$ & $\times$ & & & & & & & & & & & & & & \\
\hline 9 & $\times$ & $\times$ & $\times$ & $x$ & $x$ & & & $x$ & $x$ & $\times$ & $\times$ & & & & & & & & & & & & & \\
\hline 10 & & $\times$ & $\times$ & $x$ & $x$ & & & $x$ & $x$ & $x$ & $x$ & $x$ & $x$ & & & & & & & & & & & \\
\hline 11 & & $\times$ & $\times$ & $x$ & $x$ & $x$ & & $x$ & $x$ & $x$ & & $x$ & $x$ & $x$ & & & & & & & & & & \\
\hline 12 & & $x$ & $\times$ & $x$ & $x$ & $x$ & & $x$ & $x$ & $x$ & & $x$ & $x$ & $x$ & $x$ & & & & & & & & & \\
\hline 13 & & $x$ & $\times$ & $x$ & $x$ & $x$ & & & $x$ & $\times$ & & $x$ & $x$ & $x$ & $x$ & $x$ & $x$ & & & & & & & \\
\hline 14 & & $\times$ & $\times$ & $x$ & $\times$ & $x$ & & & $x$ & $\times$ & & $x$ & $\times$ & $x$ & $x$ & $x$ & $x$ & $x$ & & & & & & \\
\hline 15 & & $x$ & $\times$ & $x$ & $x$ & $x$ & & & $x$ & $\times$ & $\times$ & $x$ & $x$ & & $x$ & $x$ & $x$ & $x$ & $x$ & & & & & \\
\hline 16 & & $\times$ & $\times$ & $\times$ & $x$ & $x$ & & & $\times$ & $\times$ & $\times$ & $\times$ & $\times$ & & $\times$ & $\times$ & $\times$ & $\times$ & $x$ & $x$ & & & & \\
\hline 17 & & $\times$ & $x$ & $x$ & $x$ & $x$ & & & $x$ & $x$ & $x$ & $x$ & $x$ & & $x$ & $x$ & $x$ & $x$ & $x$ & $x$ & $x$ & & & \\
\hline 18 & & $\times$ & $\times$ & $\times$ & $\times$ & $\times$ & & & $\times$ & $\times$ & $\times$ & $\times$ & $\times$ & & $\times$ & $\times$ & $\times$ & $\times$ & $\times$ & $x$ & $\times$ & $\times$ & & \\
\hline 19 & & $\times$ & $\times$ & $x$ & $x$ & $x$ & & & $x$ & $x$ & $\times$ & $x$ & $x$ & & $x$ & $x$ & $x$ & $x$ & $x$ & $x$ & $x$ & $x$ & $x$ & \\
\hline 20 & & $\times$ & $\times$ & $x$ & $x$ & $x$ & & & $x$ & $\times$ & $x$ & $x$ & $x$ & & $x$ & $x$ & $x$ & $x$ & $x$ & $x$ & $x$ & $x$ & $x$ & $x$ \\
\hline
\end{tabular}


Table 9

California road network BD-PO and FRLM solution times (s) for different number of EV types between each OD pair.

\begin{tabular}{|c|c|c|c|c|c|c|c|c|c|c|c|c|c|c|c|c|}
\hline \multirow[b]{2}{*}{$\mathrm{p}$} & \multicolumn{2}{|l|}{$t=2$} & \multicolumn{2}{|l|}{$t=3$} & \multicolumn{2}{|l|}{$t=4$} & \multicolumn{2}{|l|}{$t=5$} & \multicolumn{2}{|l|}{$t=6$} & \multicolumn{2}{|l|}{$t=7$} & \multicolumn{2}{|l|}{$t=8$} & \multicolumn{2}{|l|}{$t=9$} \\
\hline & BD-PO & AC-PC & BD-PO & AC-PC & BD-PO & AC-PC & BD-PO & AC-PC & BD-PO & AC-PC & BD-PO & AC-PC & BD-PO & AC-PC & BD-PO & AC-PC \\
\hline 1 & 3.9 & 18.3 & 7.8 & 34.9 & 15.6 & 155.0 & 20.1 & 287.8 & 32.5 & 426.7 & 52.2 & 725.2 & 92.0 & 897.4 & 126.6 & $\mathrm{~N} / \mathrm{A}$ \\
\hline 2 & 5.5 & 11.9 & 15.5 & 89.7 & 25.6 & 152.7 & 41.0 & 293.1 & 48.3 & 456.6 & 107.2 & 372.2 & 151.2 & 513.1 & 256.9 & $\mathrm{~N} / \mathrm{A}$ \\
\hline 3 & 9.0 & 12.1 & 16.5 & 9.9 & 32.8 & 49.2 & 44.0 & 103.7 & 88.0 & 307.7 & 122.2 & 165.4 & 208.3 & 235.7 & 396.5 & N/A \\
\hline 4 & 7.8 & 13.0 & 25.2 & 62.7 & 33.8 & 140.5 & 68.2 & 283.9 & 90.6 & 306.3 & 202.2 & 435.8 & 274.2 & 542.5 & 516.1 & N/A \\
\hline 5 & 6.6 & 4.6 & 22.1 & 9.9 & 38.4 & 18.7 & 53.1 & 54.6 & 97.5 & 70.7 & 129.1 & 70.7 & 312.7 & 256.4 & 446.1 & $\mathrm{~N} / \mathrm{A}$ \\
\hline 6 & 7.9 & 11.0 & 22.6 & 30.8 & 42.2 & 49.5 & 84.8 & 236.2 & 95.5 & 157.9 & 225.6 & 166.1 & 401.5 & 340.5 & 460.7 & N/A \\
\hline 7 & 9.6 & 13.2 & 32.7 & 26.6 & 77.1 & 141.7 & 77.8 & 317.9 & 140.9 & 330.4 & 227.0 & 358.9 & 383.5 & 441.0 & 536.9 & N/A \\
\hline 8 & 12.7 & 15.7 & 41.7 & 46.2 & 71.4 & 213.5 & 132.3 & 358.2 & 166.7 & 570.0 & 252.3 & 497.5 & 532.9 & 483.4 & 819.1 & N/A \\
\hline 9 & 16.9 & 23.4 & 46.2 & 102.7 & 96.5 & 240.5 & 145.9 & 437.9 & 297.0 & 1389.3 & 353.4 & 564.6 & 716.8 & 690.0 & 1070.9 & N/A \\
\hline 10 & 18.5 & 16.0 & 59.1 & 67.7 & 139.5 & 175.4 & 195.3 & 685.4 & 318.3 & 830.1 & 432.1 & 715.6 & 1066.4 & 1015.5 & 1588.0 & N/A \\
\hline 11 & 22.8 & 15.2 & 93.7 & 133.2 & 137.4 & 257.9 & 251.2 & 658.8 & 294.1 & 979.7 & 450.3 & 762.9 & 918.9 & 943.1 & 1328.9 & N/A \\
\hline 12 & 32.4 & 22.4 & 137.0 & 211.8 & 170.7 & 293.4 & 328.9 & 447.7 & 517.6 & 747.7 & 627.5 & 1304.3 & 1363.2 & $\mathrm{~N} / \mathrm{A}$ & 1740.3 & N/A \\
\hline 13 & 101.5 & 47.3 & 263.7 & 392.1 & 216.5 & 314.9 & 315.2 & 585.6 & 459.2 & 1991.3 & 804.9 & 1969.9 & 1340.9 & 863.7 & 2071.6 & $\mathrm{~N} / \mathrm{A}$ \\
\hline 14 & 186.0 & 53.4 & 1140.9 & 918.2 & 203.0 & 206.8 & 336.0 & 521.1 & 670.0 & 957.2 & 942.2 & 2202.8 & 1777.9 & 1388.8 & 3573.9 & N/A \\
\hline 15 & 163.9 & 32.8 & 1360.8 & 502.4 & 240.1 & 265.1 & 623.6 & 990.9 & 872.0 & 1328.0 & 1798.9 & 5226.4 & 1791.6 & $\mathrm{~N} / \mathrm{A}$ & 2717.3 & N/A \\
\hline
\end{tabular}




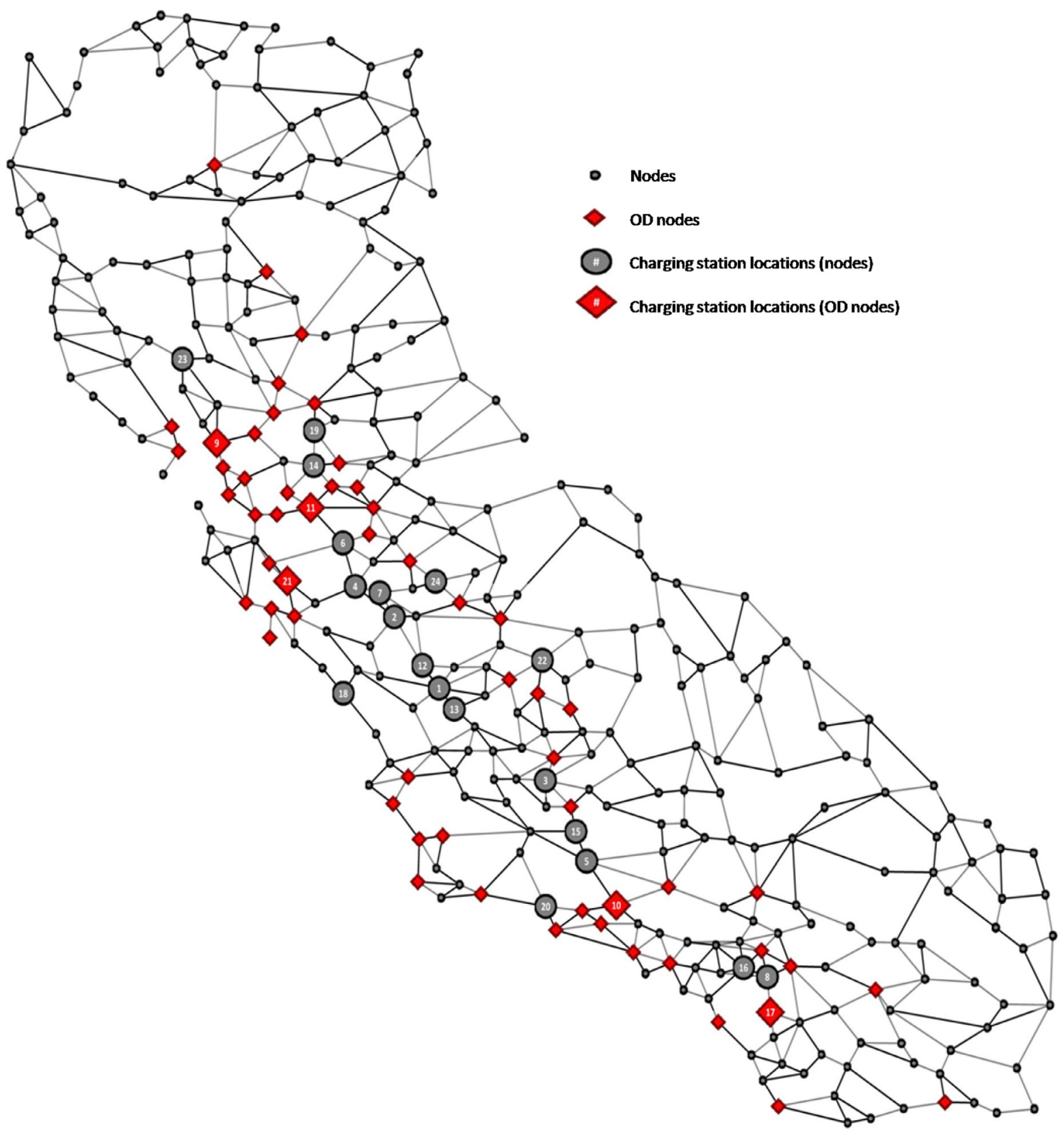

Fig. 4. Optimal charging station locations for California road network.

Table 9 shows the solution times of BD-PO method and the arc-cover path-cover (AC-PC) model by Capar et al. (2013) to solve FRLP. The first column is the number of stations $(p)$ to be located. In the second and third columns, the solution times are depicted in a network with $t=2$ different EV types between each OD pair. The results for $t \in\{2,3, \ldots, 9\}$ are also shown in the table. The BD-PO algorithm consistently solves all of the instances whereas the AC-PC fails to solve some of the large scale instances for $t=8$ and cannot solve any of the instances for $t=9$ due to excessive memory requirement. 17 of the 120 AC-PC instances could not be written to the memory of the computer. Therefore our BD-PO approach gives us the opportunity to solve large-scale instances of the problem. Furthermore, it also brings along computational efficiency. In 80 of the remaining 103 instances, BD-PO algorithm outperformed the AC-PC. Average acceleration in runtime is 1.72 times and on some instances, the BD-PO algorithm solves 14.33 times faster than the AC-PC. Figs. 5 and 6 compare the solution times of the AC-PC and the BD-PO algorithm for $p=1, \ldots, 15$ and for different EV types, respectively. Note that both figures depict the averages of those instances that both algorithms can solve to optimality. 


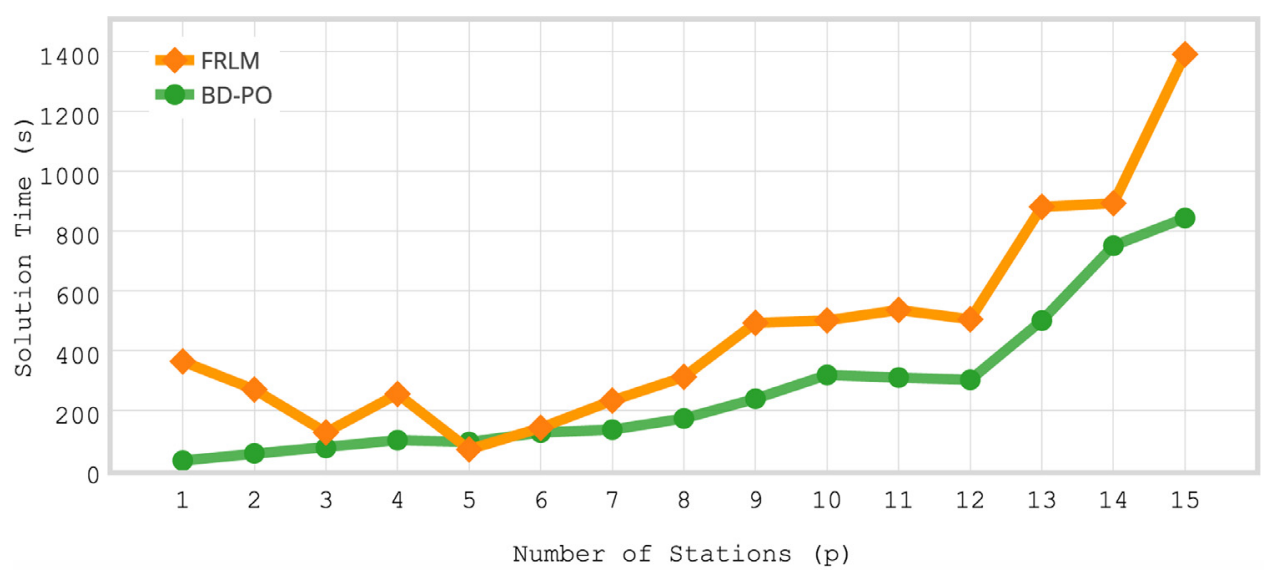

Fig. 5. Solution times of AC-PC model and BD-PO in California road network for changing number of stations.

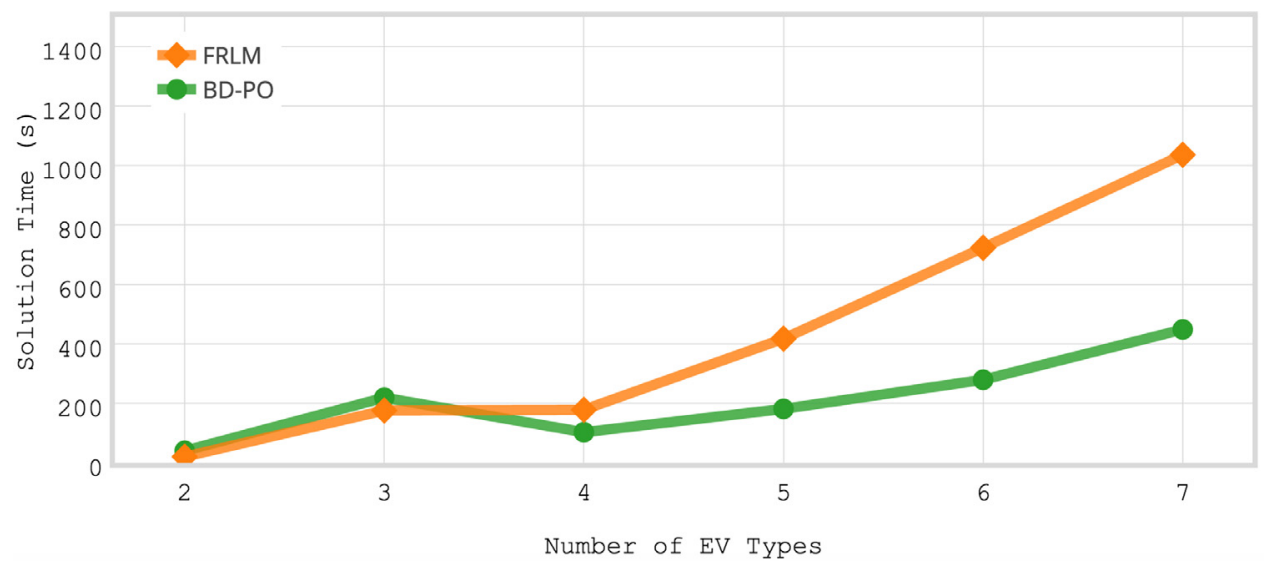

Fig. 6. Solution times of AC-PC and BD-PO in California road network for changing number of EV types.

\section{Conclusions}

Potential environmental benefits of alternative fuel vehicles (AFVs) such as electric vehicles (EVs) and plug-in hybrid electric vehicles (PHEVs) are widely discussed in the existing literature. Maximizing the vehicle flow might be tricky when it comes to maximizing the environmental benefits, because hybrid vehicles can drive on both regular gasoline and alternative forms of fuels. Even though they are not considered in location decisions, they also widely contribute to the environmental benefits and maximal utilization of alternative-fuels needs to be considered as well. Maximizing the electricity usage in transportation is also equivalent to minimizing the total cost under the current cost structure between the electricity and the gasoline.

To this end, we present the charging station location problem with plug-in hybrid electric vehicles (CSLP-PHEV) as an extension of the flow refueling location problem (FRLP). Different than previous studies, we consider hybrid vehicles in addition to single-type fueled vehicles when locating the charging stations. Rather than considering solely the vehicle flows, our objective is to maximize the exploitation of the alternative fuels. With this green viewpoint, we maximize the environmental benefits.

Additionally considering the PHEVs beside EVs complicate the already-difficult FRLP problem. In particular, we deal with this challenge by proposing an effective BD technique. Using the special structure of the formulation, we are able to construct subproblem solutions and associated cuts without the need to build and solve LPs. Furthermore, we can cleverly 'pick' the Pareto-optimal cuts among several alternative cuts. We tested alternative cut construction techniques and results indicate that Pareto-optimal cut generation effectively decreases the solution times in orders of magnitude. Computational studies show that the BD algorithm is capable of solving realistic size instances.

The scope of our problem and algorithm is not limited with PHEVs. With minor modifications, it can easily be adapted for all types of multi-fuel vehicles with engines that can run on multiple types of fuels such as Volvo Multi-Fuel, several military vehicles including M35 cargo truck and T-80 truck, or flex-fuel vehicles that run on gasoline or gasoline-ethanol 
blends of up to 85\% ethanol (U.S. Department of Energy, 2016a) such as Volvo Flexifuel, Volkswagen Total Flex, Chevrolet FlexPower, Renault Hi-Flex, and Ford Flexifuel.

Future research in this area can be directed towards considering capacities of the stations. As the number of EVs and PHEVs increase, the capacities of the stations may become critical. Furthermore, considering the deviations of the drivers from the shortest paths might be another research direction.

\section{Acknowledgments}

This work was supported by the Scientific and Technological Research Council of Turkey (TUBITAK) under the grant number 214M211. We are also thankful to anonymous referees for their constructive feedbacks in leading to the current form of this article.

\section{Appendix A. Algorithms}

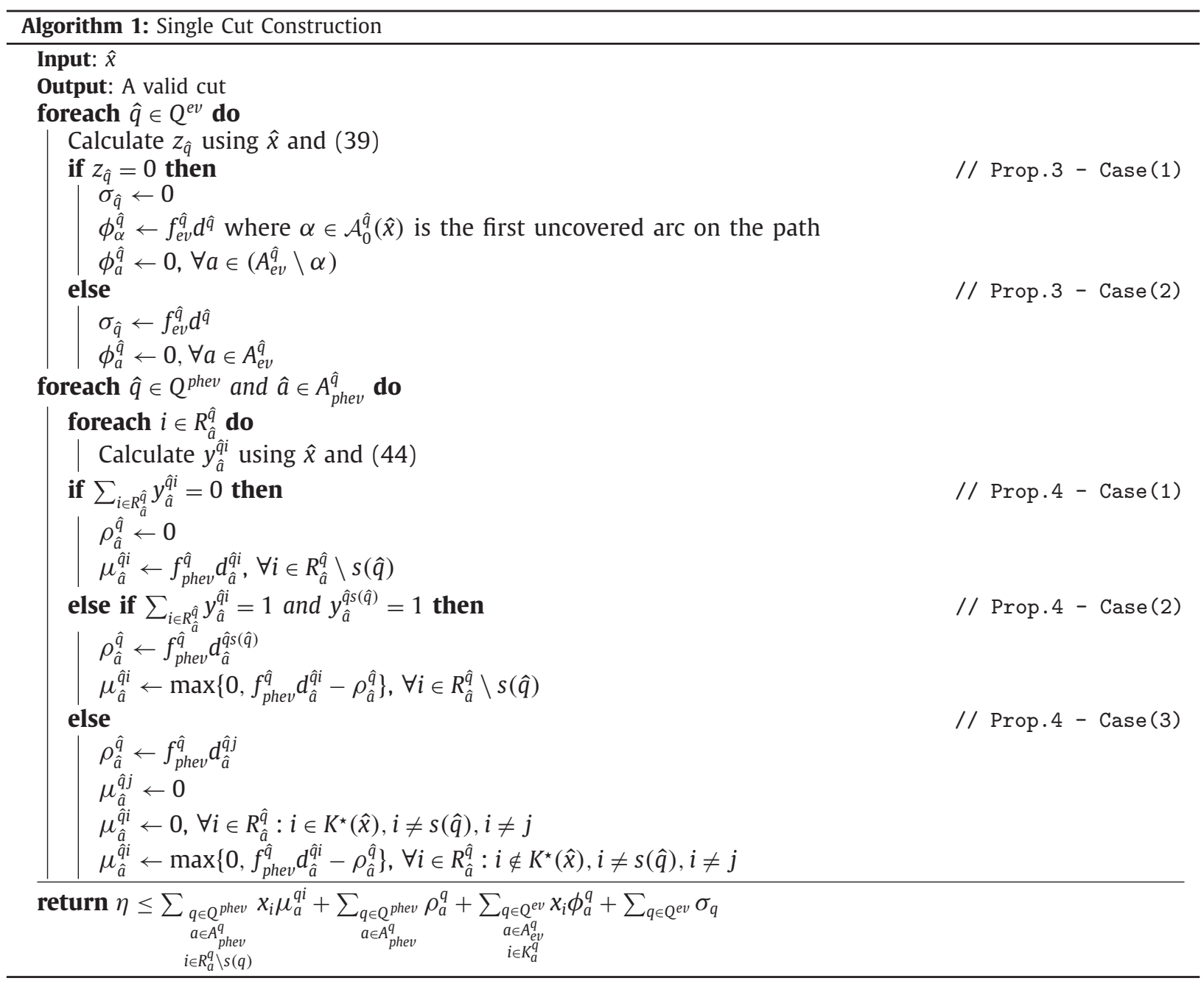




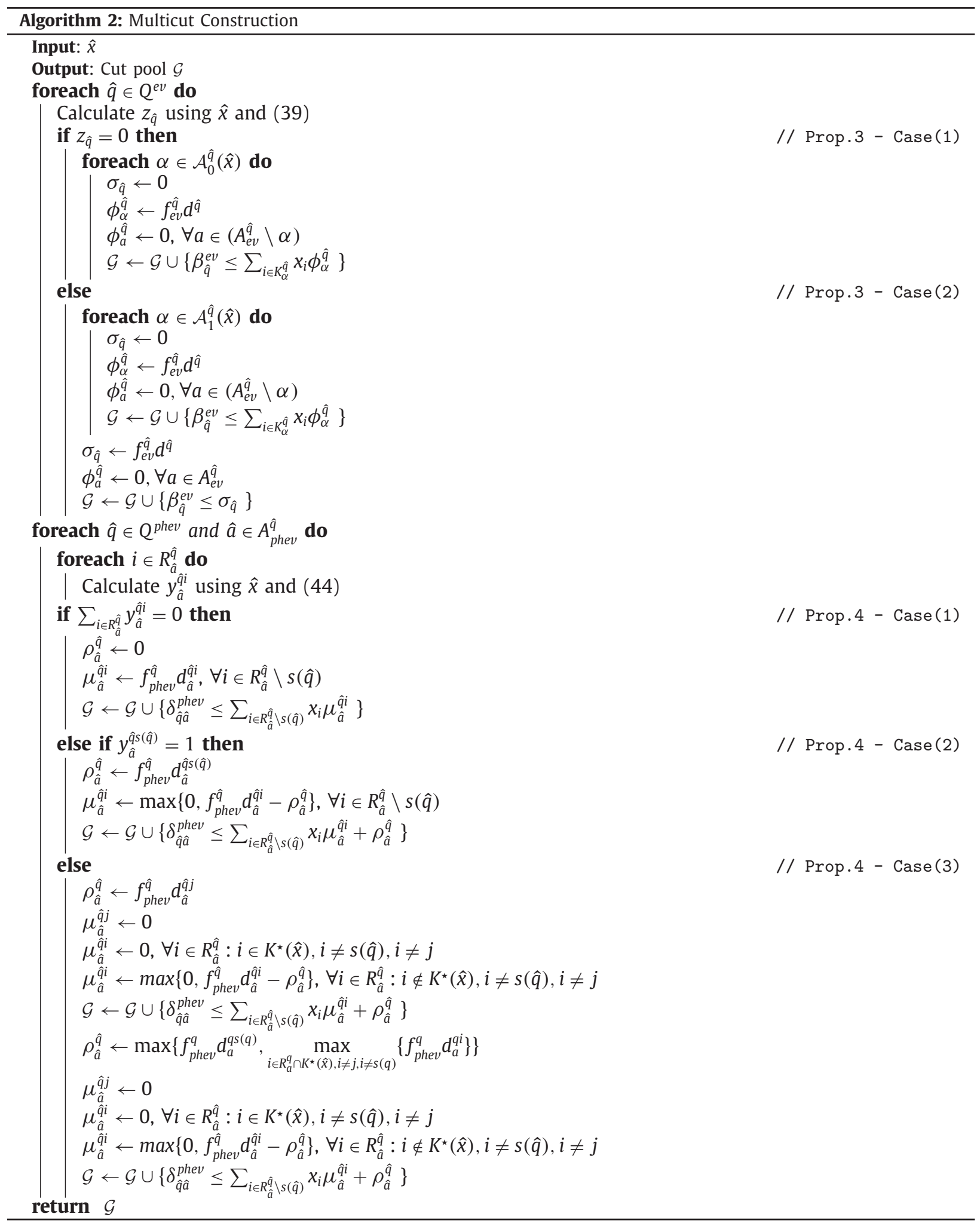




\section{References}

Arslan, O., Yıldız, B., Karașan, O.E., 2014. Impacts of battery characteristics, driver preferences and road network features on travel costs of a plug-in hybrid electric vehicle (PHEV) for long-distance trips. Energy Policy 74 (0), 168-178.

Arslan, O., Yıldız, B., Karaşan, O.E., 2015. Minimum cost path problem for plug-in hybrid electric vehicles. Transp. Res. Part E 80, $123-141$.

Axsen, J., Kurani, K.S., 2008. The early US market for PHEVs: anticipating consumer awareness, recharge potential, design priorities and energy impacts. Research Report UCD-ITS-RR-08-22. Institute of Transportation Studies, University of California, Davis.

Bapna, R., Thakur, L.S., Nair, S.K., 2002. Infrastructure development for conversion to environmentally friendly fuel. Eur. J. Oper. Res. 142 (3), $480-496$.

Benders, J.F., 1962. Partitioning procedures for solving mixed-variables programming problems. Numerische mathematik 4 (1), $238-252$.

Birge, J.R., Louveaux, F.V., 1988. A multicut algorithm for two-stage stochastic linear programs. Eur. J. Oper. Res. 34 (3), $384-392$.

de Camargo, R., Jr., G.M., Luna, H., 2008. Benders decomposition for the uncapacitated multiple allocation hub location problem. Computers \& Operations Research 35 (4), 1047-1064.

de Camargo, R.S., de Miranda Jr, G., Luna, H.P.L., 2009. Benders decomposition for hub location problems with economies of scale. Transp. Sci. 43 (1), 86-97. Capar, I., Kuby, M., 2012. An efficient formulation of the flow refueling location model for alternative-fuel stations. IIE Trans. 44 (8), $622-636$.

Capar, I., Kuby, M., Leon, V.J., Tsai, Y.-J., 2013. An arc cover path cover formulation and strategic analysis of alternative-fuel station locations. Eur. J. Oper. Res. 227 (1), 142-151.

Center for Sustainable Energy, 2015. California air resources board clean vehicle rebate project, rebate statistics. URL: http://energycenter.org/ clean-vehicle-rebate-project/rebate-statistics. Retrieved Aug 20, 2015. Data last updated Aug 17, 2015.

Chung, S.H., Kwon, C., 2015. Multi-period planning for electric car charging station locations: A case of Korean expressways. Eur. J. Oper. Res. 242 (2), 677-687. http://dx.doi.org/10.1016/j.ejor.2014.10.029.

Codato, G., Fischetti, M., 2006. Combinatorial Benders' cuts for mixed-integer linear programming. Oper. Res. 54 (4), $756-766$.

Congress, U., 2005. Energy policy act of 2005. Public Law 109 (58), 42.

Contreras, I., Cordeau, J.-F., Laporte, G., 2011. Benders decomposition for large-scale uncapacitated hub location. Oper. Res. 59 (6), 1477-1490. doi:10.1287/ opre.1110.0965.

Cordeau, J.-F., Soumis, F., Desrosiers, J., 2000. A Benders decomposition approach for the locomotive and car assignment problem. Transp. Sci. 34 (2), $133-149$.

Cordeau, J.-F., Stojković, G., Soumis, F., Desrosiers, J., 2001. Benders decomposition for simultaneous aircraft routing and crew scheduling. Transp. Sci. 35 (4), $375-388$.

Costa, A.M., 2005. A survey on Benders decomposition applied to fixed-charge network design problems. Comput. Oper. Res. 32 (6), $1429-1450$.

Erdoğan, S., Miller-Hooks, E., 2012. A green vehicle routing problem. Transp. Res. Part E 48 (1), 100-114. Select Papers from the 19th International Symposium on Transportation and Traffic Theory

Fontaine, P., Minner, S., 2014. Benders decomposition for discretecontinuous linear bilevel problems with application to traffic network design. Transp. Res. Part B 70 (0), 163-172.

Froyland, G., Maher, S.J., Wu, C.-L., 2013. The recoverable robust tail assignment problem. Transp. Sci. 48 (3), $351-372$.

He, F., Wu, D., Yin, Y., Guan, Y., 2013. Optimal deployment of public charging stations for plug-in hybrid electric vehicles. Transp. Res. Part B 47 (0), 87-101.

Hodgson, M.J., 1990. A flow-capturing location-allocation model. Geograph. Anal. 22 (3), 270-279.

Hosseini, M., MirHassani, S., 2015. Refueling-station location problem under uncertainty. Transp. Res. Part E 84, 101-116. http://dx.doi.org/10.1016/j.tre.2015. 10.009.

IBM, 2013. Ibm ilog cplex optimization studio. URL: http://www-03.ibm.com/software/products/en/ibmilogcpleoptistud/.

Irle, R., Pontes, J., Irle, V., 2016. Global plug-in vehicle sales 2015. URL: http://www.ev-volumes.com/country/total-world-plug-in-vehicle-volumes/. Accessed May 25, 2016.

Jochem, P., Brendel, C., Reuter-Oppermann, M., Fichtner, W., Nickel, S., 2015. Optimizing the allocation of fast charging infrastructure along the german autobahn. J. Bus. Econ. 1-23.

Khatami, M., Mahootchi, M., Farahani, R.Z., 2015. Benders decomposition for concurrent redesign of forward and closed-loop supply chain network with demand and return uncertainties. Transp. Res. Part E 79 (0), 1-21.

Kim, J.-G., Kuby, M., 2012. The deviation-flow refueling location model for optimizing a network of refueling stations. Int. J. Hydrogen Energy 37 (6), 5406-5420.

Kim, J.-G., Kuby, M., 2013. A network transformation heuristic approach for the deviation flow refueling location model. Comput. Oper. Res. 40 (4), 1122-1131.

Kuby, M., Lim, S., 2005. The flow-refueling location problem for alternative-fuel vehicles. Socio-Econ. Plann. Sci. 39 (2), $125-145$.

Kuby, M., Lim, S., 2007. Location of alternative-fuel stations using the flow-refueling location model and dispersion of candidate sites on arcs. Netw. Spatial Econ. 7 (2), 129-152.

Kuby, M., Lines, L., Schultz, R., Xie, Z., Kim, J.-G., Lim, S., 2009. Optimization of hydrogen stations in Florida using the flow-refueling location model. Int. J. Hydrogen Energy 34 (15), 6045-6064.

Kuby, M.J., Kelley, S.B., Schoenemann, J., 2013. Spatial refueling patterns of alternative-fuel and gasoline vehicle drivers in Los Angeles. Transp. Res. Part D $25(0), 84-92$.

Lei, C., Lin, W.-H., Miao, L., 2014. A multicut l-shaped based algorithm to solve a stochastic programming model for the mobile facility routing and scheduling problem. Eur. J. Oper. Res. 238 (3), 699-710.

Li, S., Huang, Y., 2014. Heuristic approaches for the flow-based set covering problem with deviation paths. Transp. Res. Part E 72 (0), $144-158$.

Lim, S., Kuby, M., 2010. Heuristic algorithms for siting alternative-fuel stations using the flow-refueling location model. Eur. J. Oper. Res. 204 (1), 51-61.

Lin, Z., Greene, D., 2011. Promoting the market for plug-in hybrid and battery electric vehicles: role of recharge availability. Transp. Res. Record (2252) 49-56.

Magnanti, T.L., Wong, R.T., 1981. Accelerating benders decomposition: Algorithmic enhancement and model selection criteria. Operations research 29 (3), 464-484.

Melaina, M., Bremson, J., 2008. Refueling availability for alternative fuel vehicle markets: sufficient urban station coverage. Energy Policy 36 (8), $3233-3241$.

Melaina, M.W., 2003. Initiating hydrogen infrastructures: preliminary analysis of a sufficient number of initial hydrogen stations in the us. Int. J. Hydrogen Energy 28 (7), 743-755.

MirHassani, S.A., Ebrazi, R., 2013. A flexible reformulation of the refueling station location problem. Transp. Sci. 47 (4), $617-628$.

Nie, Y.M., Ghamami, M., 2013. A corridor-centric approach to planning electric vehicle charging infrastructure. Transp. Res. Part B 57 (0), 172-190.

Papadakos, N., 2008. Practical enhancements to the Magnanti-Wong method. Oper. Res. Lett. 36 (4), $444-449$.

Pontes, J., 2016. World top 20 december 2015 special edition (updated). URL: http://ev-sales.blogspot.com/2016/01/world-top-20-december-2015-special. html Accessed May 25, 2016.

Romm, J., 2006. The car and fuel of the future. Energy Policy 34 (17), 2609-2614.

Martins de Sá, E., Contreras, I., Cordeau, J.-F., Saraiva de Camargo, R., de Miranda, G., 2015. The hub line location problem. Transp. Sci 49 (3), $500-518$.

de Sá, E.M., de Camargo, R.S., de Miranda, G., 2013. An improved Benders decomposition algorithm for the tree of hubs location problem. Eur. J. Oper. Res. 226 (2), 185-202.

Schneider, M., Stenger, A., Goeke, D., 2014. The electric vehicle-routing problem with time windows and recharging stations. Transp. Sci. 48 (4), $500-520$. doi: $10.1287 / \operatorname{trsc} .2013 .0490$. 
Shelton, S., 2016. Plug-in hybrids predicted to soon globally outsell evs. URL: http://www.hybridcars.com/plug-in-hybrids-predicted-to-outsell-evs-globallyin-near-future Accessed May 25, 2016.

Trukhanov, S., Ntaimo, L., Schaefer, A., 2010. Adaptive multicut aggregation for two-stage stochastic linear programs with recourse. Eur. J. Oper. Res. 206 (2), 395-406.

Upchurch, C., Kuby, M., Lim, S., 2009. A model for location of capacitated alternative-fuel stations. Geograph. Anal. 41 (1), 85-106.

U.S. Department of Energy, 2014. Alternative fuel vehicles in use. URL: www.afdc.energy.gov/data/10300. Accessed May 22, 2015.

U.S. Department of Energy, 2015a. Benefits and considerations of electricity as a vehicle fuel. URL: www.afdc.energy.gov/fuels/electricitybenefits.html. Accessed May 22, 2015.

U.S. Department of Energy, 2015b. Hydrogen benefits and considerations. URL: www.afdc.energy.gov/fuels/hydrogenbenefits.html Accessed May 22, 2015.

U.S. Department of Energy, 2016a. Flex-fuel vehicles. https://www.fueleconomy.gov/feg/flextech.shtml.

U.S. Department of Energy, 2016b. U.s. plug-in electric vehicle sales by model. URL:http://www.afdc.energy.gov/uploads/data/datasource/10567/ 10567pevsales.xlsx Accessed May 25, 2016.

Üster, H., Kewcharoenwong, P., 2011. Strategic design and analysis of a relay network in truckload transportation. Transp. Sci. 45 (4), $505-523$.

Walsh, K., Enz, C.A., Canina, L., 2004. The impact of gasoline price fluctuations on lodging demand for \{US\} brand hotels. Int. J. Hospitality Manage. 23 (5), 505-521. http://dx.doi.org/10.1016/j.ijhm.2004.02.004. 2003 Review Issue URL: http://www.sciencedirect.com/science/article/pii/S0278431904000222

Wang, Y.-W., 2011. Locating flow-recharging stations at tourist destinations to serve recreational travelers. Int. J. Sustainable Transp. 5 (3), $153-171$.

Wang, Y.-W., Lin, C.-C., 2009. Locating road-vehicle refueling stations. Transp. Res. Part E 45 (5), 821-829.

Wang, Y.-W., Lin, C.-C., 2013. Locating multiple types of recharging stations for battery-powered electric vehicle transport. Transp. Res. Part E 58, 76-87.

Wang, Y.-W., Wang, C.-R., 2010. Locating passenger vehicle refueling stations. Transp. Res. Part E 46 (5), 791-801.

Weis, C., Axhausen, K., Schlich, R., Zbinden, R., 2010. Models of mode choice and mobility tool ownership beyond 2008 fuel prices. Transp. Res. Rec. 2157 , 86-94.

Wheatley, D., Gzara, F., Jewkes, E., 2015. Logic-based Benders decomposition for an inventory-location problem with service constraints. Omega 55 (0), $10-23$.

Windecker, A., Ruder, A., 2013. Fuel economy, cost, and greenhouse gas results for alternative fuel vehicles in 2011. Transp. Res. Part D 23 (0), 34-40.

Wu, P., Hartman, J.C., Wilson, G.R., 2005. An integrated model and solution approach for fleet sizing with heterogeneous assets. Transp. Sci. 39 (1), 87-103.

Yıldız, B., Arslan, O., Karaşan, O.E., 2016. A branch and price approach for routing and refueling station location model. Eur. J. Oper. Res. 248 (3), 815-826.

You, F., Grossmann, I.E., 2013. Multicut Benders decomposition algorithm for process supply chain planning under uncertainty. Ann. Oper. Res. 210 (1), $191-211$. 